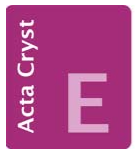

CRYSTALLOGRAPHIC COMMUNICATIONS

ISSN 2056-9890

Received 18 December 2014

Accepted 5 January 2015

Edited by M. Weil, Vienna University of Technology, Austria

Keywords: crystal structure; picolinolylhydrazone; intramolecular hydrogen bonding; ruthenium(II) polypyridyl complex.

CCDC reference: 1042209 Supporting information: this article has supporting information at journals.iucr.org/e

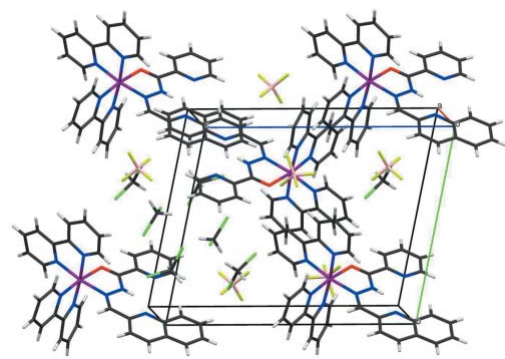

OPEN $\curvearrowright$ ACCESS

\section{Crystal structure of bis(2,2'-bipyridine) $\left[N^{\prime}\right.$-(quino- lin-2-ylmethylidene)pyridine-2-carbohydrazide]- ruthenium(II) bis(tetrafluoridoborate) dichloro- methane trisolvate}

\author{
Asami Mori, ${ }^{a}$ Takayoshi Suzuki ${ }^{\mathrm{a} *}$ and Kiyohiko Nakajima ${ }^{\mathrm{b}}$ \\ ${ }^{\mathbf{a}}$ Department of Chemistry, Faculty of Science, Okayama University, Okayama, 700-8530, Japan, and ${ }^{\mathbf{b}}$ Department of \\ Chemistry, Aichi University of Education, Kariya, Aichi 448-8542, Japan. *Correspondence e-mail: \\ suzuki@okayama-u.ac.jp
}

The title compound, $\left[\mathrm{Ru}\left(\mathrm{C}_{10} \mathrm{H}_{8} \mathrm{~N}_{2}\right)_{2}\left(\mathrm{C}_{16} \mathrm{H}_{12} \mathrm{~N}_{4} \mathrm{O}\right)\right]\left(\mathrm{BF}_{4}\right)_{2} \cdot 3 \mathrm{CH}_{2} \mathrm{Cl}_{2}$, crystallizes with one complex dication, two $\mathrm{BF}_{4}{ }^{-}$counter-anions and three dichloromethane solvent molecules in the asymmetric unit. The central $\mathrm{Ru}^{\mathrm{II}}$ atom adopts a distorted octahedral coordination sphere with two 2,2'-bipyridine (bpy) and one quinoline-2-carbaldehyde (pyridine-2-carbonyl)hydrazone $(\mathrm{H} L)$ ligand. The hydrazone ligand has a $Z$ form and coordinates to the $\mathrm{Ru}^{\mathrm{II}}$ atom via the amide-O and imine- $\mathrm{N}$ atoms, affording a planar five-membered chelate ring, while its pyridine- $\mathrm{N}$ and quinoline- $\mathrm{N}$ donor atoms in the substituents are noncoordinating. The hydrazone $\mathrm{N}-\mathrm{H}$ group forms an intramolecular hydrogen bond with the quinoline-N atom. In the crystal, the quinoline moiety of $\mathrm{H} L$ shows the shortest $\pi-\pi$ stacking interaction with the pyridine substituent of $\mathrm{H} L$ in a neighbouring complex, the centroid-to-centroid distance being 3.793 (3) $\AA$.

\section{Chemical context}

Aroylhydrazones, $\operatorname{Ar} \mathrm{C}(\mathrm{O}) \mathrm{NHN}=\mathrm{CH} R$, are easily prepared by the reaction of an aroylhydrazine $\left[A r \mathrm{C}(\mathrm{O}) \mathrm{NHNH}_{2}\right]$ with an aldehyde $(R \mathrm{CHO})$, and they can coordinate to a metal atom via the amide-O and imine- $\mathrm{N}$ atoms (Bernhardt et al., 2007; Raveendran \& Pal, 2005, 2006). These hydrazones are often obtained as a mixture of $E$ and $Z$ isomers (Su \& Aprahamian, 2014), and both isomers are generally weak acids. However, when they coordinate to a metal ion through the imine-N atom, their acidity becomes higher (Chang et al., 2010), and the deprotonated hydrazonato complexes are often isolated (Nonoyama, 1974). For example, the reaction of cis[ $\left.\mathrm{RuCl}_{2}(\text { bpy })_{2}\right]$ (bpy is 2,2'-bipyridine) and a series of aroylhydrazones in the presence of triethylamine afforded the cationic complexes $\left[\mathrm{Ru}^{\mathrm{II}}(\text { bpy })_{2}\right.$ (hydrazonato $\left.)\right]\left(\mathrm{ClO}_{4}\right.$ or $\left.\mathrm{PF}_{6}\right)$, which were unambiguously characterized by $\mathrm{X}$-ray analysis (Duan et al., 1998; Ghosh et al., 2014).

In the current study we utilized a 2-picolinoylhydrazone ( $\mathrm{Ar}$ $\left.=2-\mathrm{C}_{5} \mathrm{H}_{4} \mathrm{~N}\right)$ with a 2-quinolyl substituent on the imine- $\mathrm{C}$ atom $\left(R=2-\mathrm{C}_{9} \mathrm{H}_{6} \mathrm{~N}\right)$. This compound $(\mathrm{H} L)$ has several possible coordination modes because of the additional pyridine and quinoline ligating groups. In a previous study we investigated the reaction products from $\left[\mathrm{RuCl}_{2}\left(\mathrm{PPh}_{3}\right)_{3}\right]$ and (an $E / Z$ mixture of) $\mathrm{H} L$ under several reaction conditions, and characterized three geometrical isomers of $\left[\mathrm{RuCl}_{2}\left(\mathrm{PPh}_{3}\right)_{2}\{\mathrm{HL}\right.$ $\kappa O($ amide $), \kappa N($ imine $)\}]$ as well as a linkage isomer of $\operatorname{trans}(\mathrm{P})$ $\left[\mathrm{RuCl}_{2}\left(\mathrm{PPh}_{3}\right)_{2}\{\mathrm{HL}-\kappa N(\right.$ imine), $\kappa N($ quinoline $)\}]$ (Mori et al., 2014). Here, we have examined the reaction of the $Z$ isomer of 
$\mathrm{H} L$ and an $\mathrm{Ru}^{\mathrm{II}}(\mathrm{bpy})_{2}$ precursor prepared from cis$\left[\mathrm{RuCl}_{2}(\mathrm{bpy})_{2}\right]$ and $\mathrm{AgBF}_{4}$ (2 eq.) in ethanol. The resulting orange product had the composition $\mathrm{Ru}(\mathrm{bpy})_{2}(\mathrm{HL})\left(\mathrm{BF}_{4}\right)_{2}$, indicating the formation of an $\mathrm{Ru}^{\mathrm{II}}$ complex with a neutral hydrazone ligand, in contrast to the previous examples of $\left[\mathrm{Ru}^{\mathrm{II}}(\mathrm{bpy})_{2}\right.$ (hydrazonato) $]\left(\mathrm{ClO}_{4}\right.$ or $\left.\mathrm{PF}_{6}\right)$. Therefore, in order to determine the molecular and crystal structure of the present product, an orange prismatic crystal of the title compound, (I), $\left[\mathrm{Ru}\left(\mathrm{C}_{10} \mathrm{H}_{8} \mathrm{~N}_{2}\right)_{2}\left(\mathrm{C}_{16} \mathrm{H}_{12} \mathrm{~N}_{4} \mathrm{O}\right)\right]\left(\mathrm{BF}_{4}\right)_{2} \cdot 3 \mathrm{CH}_{2} \mathrm{Cl}_{2}$, was analysed by $\mathrm{X}$-ray diffraction.

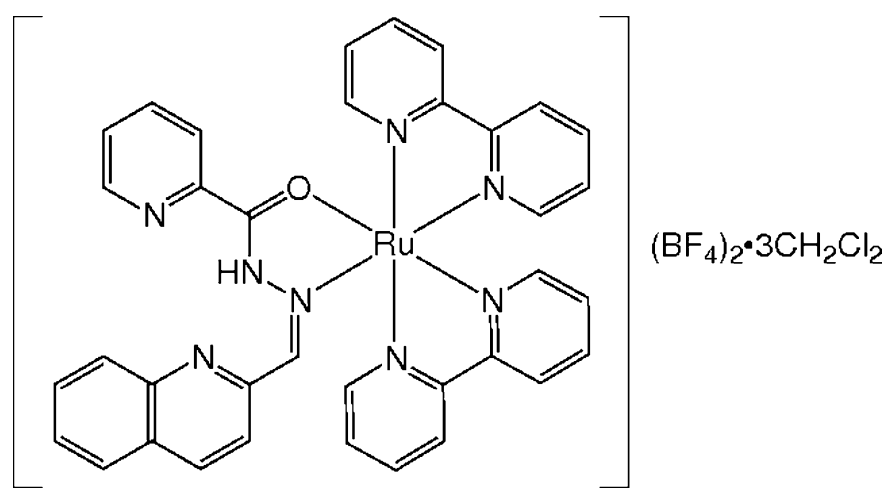

\section{Structural commentary}

The asymmetric unit of compound (I) contains one complex dication (Fig. 1), two $\mathrm{BF}_{4}{ }^{-}$counter-anions and three dichloromethane solvent molecules. In the cationic complex, the neutral hydrazone is present as its $Z$ isomer and coordinates to the $\mathrm{Ru}^{\mathrm{II}}$ atom through the amide-O and imine- $\mathrm{N}$ atoms, forming a virtually planar five-membered chelate ring [maximum deviation from the least-squares plane = $0.015(4) \AA]$, as well as two bidentate bpy co-ligands. An intramolecular hydrogen bond between the hydrazone $\mathrm{N}-\mathrm{H}$ group and the quinoline- $\mathrm{N}$ atom is observed (Table 1). The

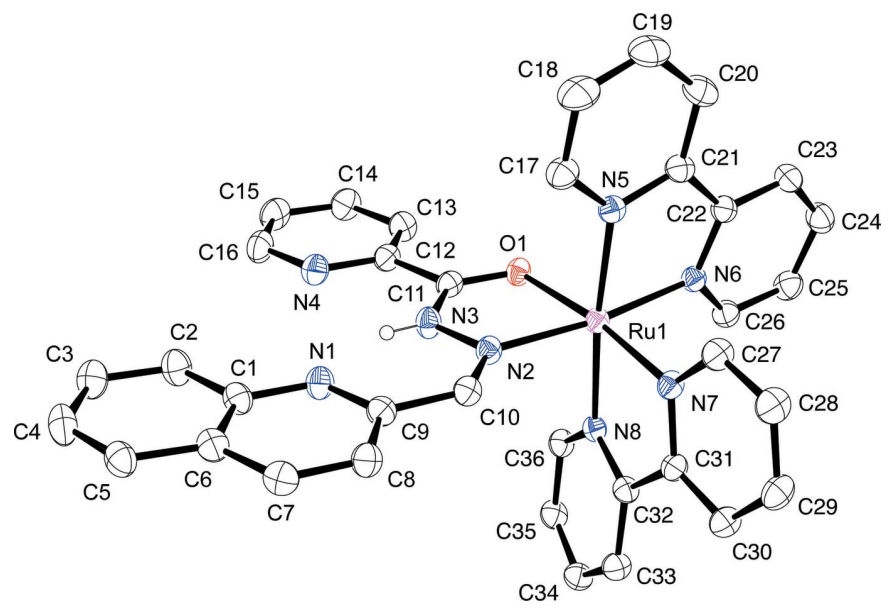

Figure 1

View of the molecular structure of the cationic complex in the title compound, showing the atom-numbering scheme, with displacement ellipsoids drawn at the $30 \%$ probability level. Hydrogen atoms except for the hydrazone $\mathrm{N}-\mathrm{H}$ group are omitted for clarity.
Table 1

Hydrogen-bond geometry $\left(\AA{ }^{\circ}\right)$.

\begin{tabular}{lllll}
\hline$D-\mathrm{H} \cdots A$ & $D-\mathrm{H}$ & $\mathrm{H} \cdots A$ & $D \cdots A$ & $D-\mathrm{H} \cdots A$ \\
\hline $\mathrm{N} 3-\mathrm{H} 1 \cdots \mathrm{N} 1$ & $0.76(6)$ & $1.90(6)$ & $2.553(6)$ & $145(6)$ \\
\hline
\end{tabular}

pyridine (py) and quinoline (qn) moieties of $\mathrm{H} L$ are noncoordinating, but their mean planes are almost co-planar to the $\mathrm{Ru}^{\mathrm{II}}$ carboxylic acid hydrazide $(\mathrm{CAH}:-\mathrm{C}(\mathrm{O}) \mathrm{NHN}=)$ chelating plane. The dihedral angles between these planes are: py $v s \mathrm{CAH}=5.4(2)$, qn $v s \mathrm{CAH}=3.7(2)$ and py $v s$ qn $=$ $2.3(2)^{\circ}$.

The Ru1-O1(amide) and Ru1-N2(imine) bond lengths in (I) are 2.090 (3) and 2.047 (4) $\AA$, respectively, which are comparable to those in $\left[\mathrm{Ru}(\text { bpy })_{2}\{3-\right.$ py $-\mathrm{C}(\mathrm{O}) \mathrm{NN}=\mathrm{CH}-$ $\left.\left.\mathrm{C}_{6} \mathrm{H}_{4}\left(4-\mathrm{NMe}_{2}\right)\right\}\right] \mathrm{ClO}_{4}[2.083$ (1) and 2.040 (1) $\mathrm{A}$, respectively; Duan et al., 1998] and [ $\mathrm{Ru}(\text { bpy })_{2}\left\{2-\mathrm{C}_{6} \mathrm{H}_{4}(\mathrm{OH})-\mathrm{C}(\mathrm{O}) \mathrm{NN}=\right.$ $\mathrm{CH}-2$-furyl $\}] \mathrm{PF}_{6} \quad[2.072(2)$ and 2.089 (1) $\AA$, respectively; Ghosh et al., 2014]. The bite angle of the hydrazone chelate, $\mathrm{O} 1-\mathrm{Ru}-\mathrm{N} 2$, in (I) is $77.8(1)^{\circ}$, which is also similar to the above-mentioned hydrazonato complexes, $78.0(1)$ and $78.6(1)^{\circ}$, respectively. Thus, the substituent groups on the carbonyl- $\mathrm{C}$ and the imine-C atoms of the aroylhydrazones, as well as the protonation (or deprotonation) states of the hydrazone $\mathrm{N}-\mathrm{H}$ moiety, do not significantly affect the structural parameters of the $\mathrm{Ru}^{\mathrm{II}}-$ (hydrazone/hydrazonate) coordination bonds.

\section{Supramolecular features}

In the crystal structure of (I) there are no remarkable hydrogen-bonding interactions between the cationic complex, $\mathrm{BF}_{4}{ }^{-}$anions and the solvated dichloromethane molecules. However, each of the planar $\mathrm{H} L$ and two bpy ligands in the

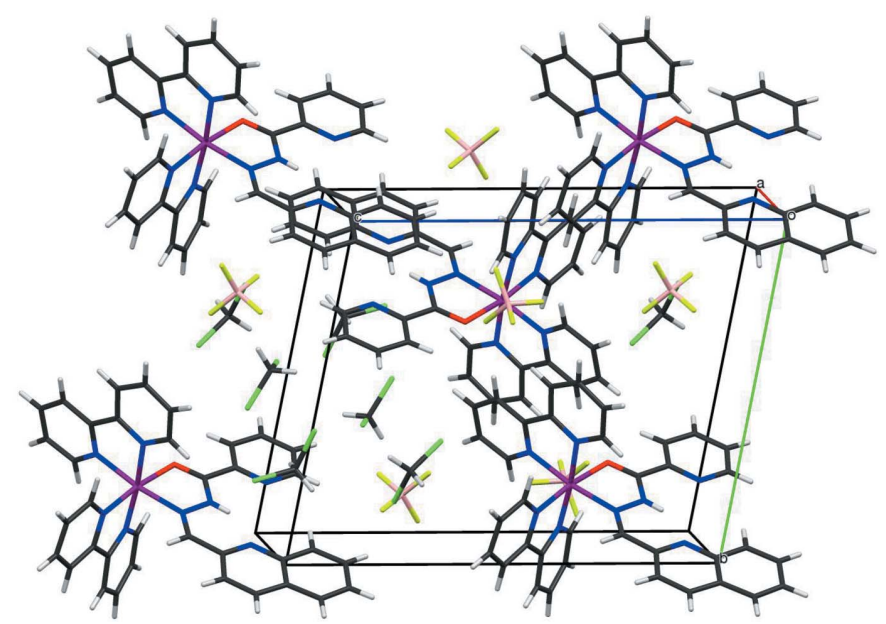

Figure 2

View of the crystal packing of the title compound, illustrating three $\pi-\pi$ stacking interactions between the complex cations. Colour code: $\mathrm{Ru}$, purple; Cl, green; F, yellow-green; O, red; N, blue; C, black; B, pink; H, grey. 
complex cation shows a $\pi-\pi$ stacking interaction with the respective neighbouring complexes (Fig. 2). The quinoline plane $(\mathrm{N} 1 / \mathrm{C} 1-\mathrm{C} 9)$ has a stacking interaction with the pyridine plane $\left(\mathrm{N} 4^{\mathrm{i}} / \mathrm{C} 12^{\mathrm{i}}-\mathrm{C} 16^{\mathrm{i}}\right)$ of $\mathrm{H} L$ in a neighbouring complex [symmetry code: (i) $-x,-y+1,-z+2$ ]; the shortest C $\cdots \mathrm{C}$ distance between these rings is $\mathrm{C} 6 \cdots \mathrm{C} 16^{\mathrm{i}}=3.444(8) \AA$ and the centroid-to-centroid distance between the planes $\mathrm{C} 1-\mathrm{C} 6$ and $\mathrm{N}^{\mathrm{i}} / \mathrm{C} 12^{\mathrm{i}}-\mathrm{C} 16^{\mathrm{i}}$ is 3.793 (3) $\AA$. One of the bpy ligands, N5/ C17-C26/N6, is stacked with the same symmetry-related ligand, $\mathrm{N} 5^{\mathrm{ii} /} / \mathrm{C} 17^{\mathrm{ii}}-\mathrm{C} 26^{\mathrm{ii}} / \mathrm{N} 6^{\mathrm{ii}}$, in a neighbouring complex [symmetry code: (ii) $-x,-y+1,-z+1$ ]; the shortest $\mathrm{C} \cdots \mathrm{C}$ distance between them is $\mathrm{C} 20 \cdots \mathrm{C} 25^{\mathrm{i}}=3.373$ (8) $\AA$, and the centroid-to-centroid distance between the N6/C22-C26 and $\mathrm{N} 6{ }^{\mathrm{ii}} / \mathrm{C} 22^{\mathrm{ii}}-\mathrm{C} 26^{\mathrm{ii}}$ planes is 3.864 (3) $\AA$. For the other bpy ligand, $\mathrm{N} 7 / \mathrm{C} 27-\mathrm{C} 36 / \mathrm{N} 8$, a similar interaction is observed, and the shortest $\mathrm{C} \cdots \mathrm{C}$ distance between them is $\mathrm{C} 32 \cdots \mathrm{C} 35^{\mathrm{iii}}=$ 3.509 (8) $\AA$ and the centroid-to-centroid distance between planes $\mathrm{N} 8 / \mathrm{C} 32-\mathrm{C} 36$ and $\mathrm{N} 8^{\mathrm{iii}} / \mathrm{C} 32^{\mathrm{iii}}-\mathrm{C} 36^{\mathrm{iii}}$ is 3.918 (3) $\AA$ [symmetry code: (iii) $-x,-y,-z+1$ ]. Considering these stacking interactions, the complex cations are arranged in a three-dimensional extended structure in the crystal.

\section{Database survey}

Four geometrical and linkage isomers of $\left[\mathrm{RuCl}_{2}\left(\mathrm{PPh}_{3}\right)_{2}(\mathrm{H} L)\right]$ with the same picolinoylhydrazone ligand, $\mathrm{H} L$, have been reported previously (Mori et al., 2014). There is no record of any $\left[\mathrm{Ru}^{\mathrm{II}}(\mathrm{bpy})_{2} \text { (carbonylhydrazone) }\right]^{2+}$ complexes with its protonated (neutral) hydrazone form in the CSD database (Version 5.35, last update May 2014; Groom \& Allen, 2014). The deprotonated (anionic) hydrazonate analogues, $\left[\mathrm{Ru}(\text { bpy })_{2}\left\{3-\mathrm{py}-\mathrm{C}(\mathrm{O}) \mathrm{NN}=\mathrm{CHC}_{6} \mathrm{H}_{4}\left(4-\mathrm{NMe}_{2}\right)\right\}\right] \mathrm{ClO}_{4}$ (Duan et al., 1998) and $\left[\mathrm{Ru}(\text { bpy })_{2}\left\{2-\mathrm{C}_{6} \mathrm{H}_{4}(\mathrm{OH})-\mathrm{C}(\mathrm{O}) \mathrm{NN}=\mathrm{CH}-2\right.\right.$-fur$\mathrm{yl}\}] \mathrm{PF}_{6}$ as well as the thiophene analogue have been reported (Ghosh et al., 2014). The structurally related compound $\left[\mathrm{Ru}^{\mathrm{II}}(\mathrm{bpy})_{2}\left\{\mathrm{C}_{6} \mathrm{H}_{5} \mathrm{C}(\mathrm{O}) \mathrm{NNC}_{6} \mathrm{H}_{5}\right\}\right] \mathrm{PF}_{6}$ with a monoanionic (radical) ligand has also been reported (Ehret et al., 2012).

\section{Synthesis and crystallization}

All reagents and solvents were commercially available and used without further purification. The starting ruthenium(II) complex, cis-[ $\left[\mathrm{RuCl}_{2}(\mathrm{bpy})_{2}\right] \cdot 2 \mathrm{H}_{2} \mathrm{O}$ (Sullivan et al., 1978), and hydrazone ligand, Z-HL (Mori et al., 2014), were prepared according to literature procedures. A mixture of cis$\left[\mathrm{RuCl}_{2}(\mathrm{bpy})_{2}\right] \cdot 2 \mathrm{H}_{2} \mathrm{O} \quad(618 \mathrm{mg}, \quad 1.19 \mathrm{mmol})$ and $\mathrm{AgBF}_{4}$ $(463 \mathrm{mg}, 2.38 \mathrm{mmol})$ in ethanol $(80 \mathrm{ml})$ was stirred in the dark at room temperature overnight. The resulting white precipitate $(\mathrm{AgCl})$ was filtered off, and $Z$ - $\mathrm{HL}(328 \mathrm{mg}, 1.19 \mathrm{mmol})$ was added to the filtrate. The mixture was heated to reflux for $9 \mathrm{~h}$ and then cooled to room temperature. The solution was concentrated to $c a .10 \mathrm{ml}$ under reduced pressure, and the resulting microcrystalline powder was collected by filtration and dried in air. Yield: $869 \mathrm{mg}(81 \%)$. Analysis calculated for $\mathrm{C}_{36} \mathrm{H}_{28} \mathrm{~B}_{2} \mathrm{~F}_{8} \mathrm{~N}_{8} \mathrm{ORu} \cdot 2 \mathrm{H}_{2} \mathrm{O}: \mathrm{C} 48.08, \mathrm{H} 3.59, \mathrm{~N} 12.46 \%$. Found: C 48.11, H 3.42, N $12.18 \%$. Orange prismatic crystals of (I)
Table 2

Experimental details.

\begin{tabular}{|c|c|}
\hline \multicolumn{2}{|l|}{ Crystal data } \\
\hline Chemical formula & $\begin{array}{l}{\left[\mathrm{Ru}\left(\mathrm{C}_{10} \mathrm{H}_{8} \mathrm{~N}_{2}\right)_{2}\left(\mathrm{C}_{16} \mathrm{H}_{12} \mathrm{~N}_{2}\right)\right]\left(\mathrm{BF}_{4}\right)_{2} \cdot .} \\
\quad 3 \mathrm{CH}_{2} \mathrm{Cl}_{2}\end{array}$ \\
\hline$M_{\mathrm{r}}$ & 1118.13 \\
\hline Crystal system, space group & Triclinic, $P \overline{1}$ \\
\hline Temperature (K) & 192 \\
\hline$a, b, c(\AA)$ & $\begin{array}{l}11.0165(12), 13.2508(15), \\
16.4285(19)\end{array}$ \\
\hline$\alpha, \beta, \gamma\left({ }^{\circ}\right)$ & $77.812(4), 76.924$ (4), 88.367 (4) \\
\hline$V\left(\AA^{3}\right)$ & $2282.9(4)$ \\
\hline$Z$ & 2 \\
\hline Radiation type & Мо $K \alpha$ \\
\hline$\mu\left(\mathrm{mm}^{-1}\right)$ & 0.77 \\
\hline Crystal size $(\mathrm{mm})$ & $0.40 \times 0.30 \times 0.25$ \\
\hline \multicolumn{2}{|l|}{ Data collection } \\
\hline Diffractometer & Rigaku R-AXIS RAPID \\
\hline Absorption correction & $\begin{array}{l}\text { Numerical (NUMABS; Rigaku, } \\
\text { 1999) }\end{array}$ \\
\hline$T_{\min }, T_{\max }$ & $0.658,0.825$ \\
\hline $\begin{array}{l}\text { No. of measured, independent and } \\
\text { observed }[I>2 \sigma(I)] \text { reflections }\end{array}$ & $22472,10378,8147$ \\
\hline$R_{\text {int }}$ & 0.076 \\
\hline$(\sin \theta / \lambda)_{\max }\left(\AA^{-1}\right)$ & 0.649 \\
\hline \multicolumn{2}{|l|}{ Refinement } \\
\hline$R\left[F^{2}>2 \sigma\left(F^{2}\right)\right], w R\left(F^{2}\right), S$ & $0.069,0.208,1.04$ \\
\hline No. of reflections & 10378 \\
\hline No. of parameters & 589 \\
\hline $\mathrm{H}$-atom treatment & $\begin{array}{l}\mathrm{H} \text { atoms treated by a mixture of } \\
\text { independent and constrained } \\
\text { refinement }\end{array}$ \\
\hline$\Delta \rho_{\max }, \Delta \rho_{\min }\left(\mathrm{e} \AA^{-3}\right)$ & $1.49,-1.02$ \\
\hline
\end{tabular}

Computer programs: RAPID-AUTO (Rigaku, 2006), CrystalStructure (Rigaku, 2010), SIR2004 (Burla et al., 2005), SHELXL2013 (Sheldrick, 2008) and ORTEP-3 for Windows (Farrugia, 2012).

suitable for X-ray analysis were obtained by diffusion of layered hexane into a dichloromethane solution.

\section{Refinement}

Crystal data, data collection and structure refinement details are summarized in Table 2. The position of the hydrazone $(\mathrm{N}-) \mathrm{H}$ atom was located in a difference Fourier map and refined with $U_{\text {iso }}=1.2 U_{\text {eq }}(\mathrm{N})$. All other $\mathrm{H}$ atoms were refined using a riding model, with $\mathrm{C}-\mathrm{H}=0.95$ (aromatic) or 0.99 (methylene) $\AA$ and $U_{\text {iso }}=1.2 U_{\text {eq }}(\mathrm{C})$.

\section{Acknowledgements}

This work was partly supported by a Grant-in-Aid for Scientific Research [Nos. 25410070 (to TS) and 24550076 (to KN)] from the Ministry of Education, Culture, Sports, Science, and Technology, Japan.

\section{References}

Bernhardt, P. V., Chin, P., Sharpe, P. C. \& Richardson, D. R. (2007). Dalton Trans. pp. 3232-3244.

Burla, M. C., Caliandro, R., Camalli, M., Carrozzini, B., Cascarano, G. L., De Caro, L., Giacovazzo, C., Polidori, G. \& Spagna, R. (2005). J. Appl. Cryst. 38, 381-388. 
Chang, M., Horiki, H., Nakajima, K., Kobayashi, A., Chang, H.-C. \& Kato, M. (2010). Bull. Chem. Soc. Jpn, 83, 905-910.

Duan, C.-Y., Lu, Z.-L., Wu, D.-B. \& You, X.-Z. (1998). Transition Met. Chem. 23, 631-634.

Ehret, F., Bubrin, M., Hübner, R., Schweinfurth, D., Hartenbach, I., Záliš, S. \& Kaim, W. (2012). Inorg. Chem. 51, 6237-6244.

Farrugia, L. J. (2012). J. Appl. Cryst. 45, 849-854.

Ghosh, B., Naskar, S., Naskar, S., Espinosa, A., Hau, S. C. K., Mak, T. C. W., Sekiya, R., Kuroda, R. \& Chattopadhyay, S. K. (2014). Polyhedron, 72, 115-121.

Groom, C. R. \& Allen, F. H. (2014). Angew. Chem. Int. Ed. 53, 662671.
Mori, A., Suzuki, T., Sunatsuki, Y., Kobayashi, A., Kato, M. \& Nakajima, K. (2014). Eur. J. Inorg. Chem. pp. 186-197.

Nonoyama, M. (1974). Inorg. Chim. Acta, 10, 133-137.

Raveendran, R. \& Pal, S. (2005). Polyhedron, 24, 57-63.

Raveendran, R. \& Pal, S. (2006). Inorg. Chim. Acta, 359, 3212-3220.

Rigaku (1999). NUMABS. Rigaku Corporation, Tokyo, Japan.

Rigaku (2006). RAPID-AUTO. Rigaku Corporation, Tokyo, Japan.

Rigaku (2010). CrystalStructure. Rigaku Corporation, Tokyo, Japan.

Sheldrick, G. M. (2008). Acta Cryst. A64, 112-122.

Su, X. \& Aprahamian, I. (2014). Chem. Soc. Rev. 43, 1963-1981.

Sullivan, B. P., Salmon, D. J. \& Meyer, T. J. (1978). Inorg. Chem. 17, 3334-3341. 


\section{supporting information}

Acta Cryst. (2015). E71, 142-145 [doi:10.1107/S2056989015000122]

\section{Crystal structure of bis(2,2'-bipyridine) [ $N^{\prime}$-(quinolin-2-ylmethyl- idene)pyridine-2-carbohydrazide]ruthenium(II) bis(tetrafluoridoborate) di- chloromethane trisolvate}

\section{Asami Mori, Takayoshi Suzuki and Kiyohiko Nakajima}

\section{Computing details}

Data collection: RAPID-AUTO (Rigaku, 2006); cell refinement: RAPID-AUTO (Rigaku, 2006); data reduction: CrystalStructure (Rigaku, 2010); program(s) used to solve structure: SIR2004 (Burla et al., 2005); program(s) used to refine structure: SHELXL2013 (Sheldrick, 2008); molecular graphics: ORTEP-3 for Windows (Farrugia, 2012); software used to prepare material for publication: SHELXL2013 (Sheldrick, 2008).

Bis(2,2'-bipyridine) [N'-(quinolin-2-ylmethylidene)pyridine-2-carbohydrazide]ruthenium(II) bis(tetrafluoridoborate) dichloromethane trisolvate

Crystal data

$\left[\mathrm{Ru}\left(\mathrm{C}_{10} \mathrm{H}_{8} \mathrm{~N}_{2}\right)_{2}\left(\mathrm{C}_{16} \mathrm{H}_{12} \mathrm{~N}_{2}\right)\right]\left(\mathrm{BF}_{4}\right)_{2} \cdot 3 \mathrm{CH}_{2} \mathrm{Cl}_{2}$

$M_{r}=1118.13$

Triclinic, $P \overline{1}$

$a=11.0165(12) \AA$

$b=13.2508(15) \AA$

$c=16.4285(19) \AA$

$\alpha=77.812(4)^{\circ}$

$\beta=76.924(4)^{\circ}$

$\gamma=88.367(4)^{\circ}$

$V=2282.9(4) \AA^{3}$

\section{Data collection}

Rigaku R-AXIS RAPID diffractometer

Detector resolution: 10.000 pixels $\mathrm{mm}^{-1}$ $\omega$ scans

Absorption correction: numerical (NUMABS; Rigaku, 1999)

$T_{\text {min }}=0.658, T_{\text {max }}=0.825$

22472 measured reflections

\section{Refinement}

Refinement on $F^{2}$

Least-squares matrix: full

$R\left[F^{2}>2 \sigma\left(F^{2}\right)\right]=0.069$

$w R\left(F^{2}\right)=0.208$

$S=1.04$

10378 reflections
$Z=2$

$F(000)=1120$

$D_{\mathrm{x}}=1.627 \mathrm{Mg} \mathrm{m}^{-3}$

Mo $K \alpha$ radiation, $\lambda=0.71075 \AA$

Cell parameters from 15690 reflections

$\theta=3.0-27.6^{\circ}$

$\mu=0.77 \mathrm{~mm}^{-1}$

$T=192 \mathrm{~K}$

Prism, orange

$0.40 \times 0.30 \times 0.25 \mathrm{~mm}$

10378 independent reflections

8147 reflections with $I>2 \sigma(I)$

$R_{\text {int }}=0.076$

$\theta_{\max }=27.5^{\circ}, \theta_{\min }=3.0^{\circ}$

$h=-14 \rightarrow 13$

$k=-16 \rightarrow 17$

$l=-21 \rightarrow 21$

589 parameters

0 restraints

Primary atom site location: structure-invariant direct methods

Secondary atom site location: difference Fourier map 
Hydrogen site location: inferred from neighbouring sites

$\mathrm{H}$ atoms treated by a mixture of independent and constrained refinement

$$
\begin{aligned}
& w=1 /\left[\sigma^{2}\left(F_{\mathrm{o}}^{2}\right)+(0.1064 P)^{2}+2.2063 P\right] \\
& \text { where } P=\left(F_{\mathrm{o}}^{2}+2 F_{\mathrm{c}}{ }^{2}\right) / 3 \\
& (\Delta / \sigma)_{\max }=0.001 \\
& \Delta \rho_{\max }=1.49 \mathrm{e} \AA^{-3} \\
& \Delta \rho_{\min }=-1.02 \mathrm{e} \AA^{-3}
\end{aligned}
$$

Special details

Experimental. ${ }^{1} \mathrm{H}$ NMR $\left(600 \mathrm{MHz}, 22{ }^{\circ} \mathrm{C}, \mathrm{CD}_{3} \mathrm{CN}\right): \delta=9.08(\mathrm{~d}, J=4.5 \mathrm{~Hz}, 1 \mathrm{H}), 8.84(\mathrm{~d}, J=5.6 \mathrm{~Hz}, 1 \mathrm{H}), 8.61(\mathrm{~d}, J=$ $5.6 \mathrm{~Hz}, 1 \mathrm{H}), 8.58(\mathrm{~d}, J=8.2 \mathrm{~Hz}, 2 \mathrm{H}), 8.56(\mathrm{~d}, J=8.7 \mathrm{~Hz}, 1 \mathrm{H}), 8.52(\mathrm{~d}, J=8.1 \mathrm{~Hz}, 1 \mathrm{H}), 8.48(\mathrm{~d}, J=7.8 \mathrm{~Hz}, 1 \mathrm{H}), 8.48(\mathrm{~d}$, $J=8.6 \mathrm{~Hz}, 1 \mathrm{H}), 8.19(\mathrm{td}, J=5.4,7.6 \mathrm{~Hz}, 2 \mathrm{H}), 8.13(\mathrm{~d}, J=7.8 \mathrm{~Hz}, 1 \mathrm{H}), 8.08-8.04(\mathrm{~m}, 4 \mathrm{H}), 8.01(\mathrm{td}, J=5.3,1.3 \mathrm{~Hz}, 1 \mathrm{H})$, $7.83(\mathrm{t}, J=7.6 \mathrm{~Hz}, 1 \mathrm{H}), 7.79-7.77(\mathrm{~m}, 2 \mathrm{H}), 7.74(\mathrm{~d}, J=6.4 \mathrm{~Hz}, 1 \mathrm{H}), 7.65(\mathrm{ddd}, J=5.0,3.9,1.4 \mathrm{~Hz}, 1 \mathrm{H}), 7.60(\mathrm{ddd}, J=$ 5.0, 3.9, $1.4 \mathrm{~Hz}, 1 \mathrm{H}), 7.58(\mathrm{~d}, J=8.7 \mathrm{~Hz}, 1 \mathrm{H}), 7.46$ (s, azomethine-H, 1H), 7.39 (ddd, J= 5.0, 4.0, $1.3 \mathrm{~Hz}, 1 \mathrm{H}), 7.33$ (ddd, $J=4.5,3.9,1.5 \mathrm{~Hz}, 1 \mathrm{H})$ p.p.m.. UV-vis (in $\left.\mathrm{CH}_{3} \mathrm{CN}\right)\left\{\lambda_{\max } / \mathrm{nm}\left(\varepsilon_{\max } M^{-1} \mathrm{~cm}^{-1}\right)\right\}: 488(16600), 332(18000), 287$ (62200), 237 (36700). Cyclic voltammetry $\left(\mathrm{CH}_{3} \mathrm{CN}\right.$ with $\left.0.1 M \mathrm{Bu}_{4} \mathrm{NClO}_{4}\right)\left\{E_{1 / 2} / \mathrm{V} v s . \mathrm{Fc}^{+} / \mathrm{Fc}(\Delta E / \mathrm{mV})\right.$ assignment $\}: 0.79$ (80) $\mathrm{Ru}^{\mathrm{III}} / \mathrm{Ru}^{\mathrm{II}},-1.04$ (72) bpy/bpy,-1.64 (69) bpy/bpy .

Geometry. All e.s.d.'s (except the e.s.d. in the dihedral angle between two 1.s. planes) are estimated using the full

\begin{tabular}{|c|c|c|c|c|}
\hline & $x$ & $y$ & $z$ & $U_{\text {iso }} * / U_{\text {eq }}$ \\
\hline Ru1 & $0.13367(3)$ & $0.24405(3)$ & $0.60979(2)$ & $0.03128(14)$ \\
\hline $\mathrm{Cl1}$ & $0.3771(2)$ & 0.4243 (2) & $0.9729(2)$ & $0.1164(9)$ \\
\hline $\mathrm{Cl} 2$ & $0.4195(2)$ & $0.28556(19)$ & $0.85446(17)$ & $0.1000(7)$ \\
\hline $\mathrm{Cl} 3$ & -0.0761 & $0.5854(4)$ & $0.8926(3)$ & 0.1842 (19) \\
\hline $\mathrm{Cl} 4$ & $0.1269(3)$ & $0.4703(3)$ & $0.8292(2)$ & $0.1411(12)$ \\
\hline $\mathrm{Cl} 5$ & $0.3573(4)$ & $0.3917(3)$ & $0.2711(2)$ & $0.1552(15)$ \\
\hline $\mathrm{Cl} 6$ & $0.3391(3)$ & 0.2153 & $0.1932(3)$ & 0.1427 (12) \\
\hline $\mathrm{F} 1$ & $0.5184(4)$ & $0.2510(4)$ & $0.6277(3)$ & 0.0938 (14) \\
\hline $\mathrm{F} 2$ & $0.6920(4)$ & $0.2642(3)$ & $0.6752(3)$ & 0.0857 (13) \\
\hline F3 & $0.6154(4)$ & $0.1074(3)$ & 0.6818 & $0.0758(11)$ \\
\hline F4 & $0.7026(4)$ & 0.2090 & $0.5556(2)$ & $0.0735(10)$ \\
\hline F5 & $0.7015(4)$ & 0.2350 & 0.1999 & 0.0807 (12) \\
\hline F6 & $0.7975(6)$ & 0.2465 & 0.3049 & $0.0990(16)$ \\
\hline F7 & $0.7015(3)$ & 0.0997 (3) & $0.3094(2)$ & $0.0667(9)$ \\
\hline F8 & $0.8746(4)$ & $0.1479(5)$ & 0.2093 & 0.1044 (17) \\
\hline O1 & -0.0113 & $0.2934(2)$ & $0.69791(18)$ & $0.0355(6)$ \\
\hline N1 & 0.1994 (4) & $0.0784(3)$ & $0.8936(2)$ & $0.0405(9)$ \\
\hline N2 & $0.1737(4)$ & $0.1702(3)$ & $0.7231(2)$ & $0.0346(8)$ \\
\hline N3 & $0.0834(4)$ & 0.1913 & $0.7909(2)$ & $0.0391(9)$ \\
\hline N4 & -0.0749 (4) & 0.2339 & $0.9244(3)$ & $0.0455(9)$ \\
\hline N5 & $0.2334(3)$ & 0.3775 & $0.5961(2)$ & $0.0339(8)$ \\
\hline N6 & 0.0848 & 0.3335 & $0.5041(2)$ & $0.0334(7)$ \\
\hline N7 & 0.2688 & 0.1771 & $0.5329(2)$ & $0.0341(8)$ \\
\hline N8 & $0.0439(3)$ & $0.1104(3)$ & $0.6106(2)$ & $0.0317(7)$ \\
\hline $\mathrm{C} 1$ & $0.2168(5)$ & $0.0357(4)$ & $0.9730(3)$ & $0.0412(10)$ \\
\hline $\mathrm{C} 2$ & $0.1309(5)$ & $0.0572(4)$ & $1.0445(3)$ & 0.0499 (12) \\
\hline $\mathrm{H} 2$ & 0.0612 & 0.0990 & 1.0369 & $0.060^{*}$ \\
\hline
\end{tabular}
covariance matrix. The cell e.s.d.'s are taken into account individually in the estimation of e.s.d.'s in distances, angles and torsion angles; correlations between e.s.d.'s in cell parameters are only used when they are defined by crystal symmetry. An approximate (isotropic) treatment of cell e.s.d.'s is used for estimating e.s.d.'s involving l.s. planes.

Fractional atomic coordinates and isotropic or equivalent isotropic displacement parameters $\left(\AA^{2}\right)$ 


\begin{tabular}{|c|c|c|c|c|}
\hline $\mathrm{C} 3$ & $0.1478(6)$ & $0.0181(5)$ & $1.1250(3)$ & 0.0555 (14) \\
\hline $\mathrm{H} 3$ & 0.0900 & 0.0328 & 1.1733 & $0.067 *$ \\
\hline $\mathrm{C} 4$ & $0.2513(6)$ & $-0.0441(5)$ & $1.1362(3)$ & $0.0542(13)$ \\
\hline $\mathrm{H} 4$ & 0.2632 & -0.0701 & 1.1923 & $0.065^{*}$ \\
\hline $\mathrm{C} 5$ & $0.3334(5)$ & -0.0673 (4) & $1.0690(3)$ & 0.0464 (11) \\
\hline H5 & 0.4007 & -0.1114 & 1.0783 & $0.056^{*}$ \\
\hline C6 & $0.3207(5)$ & -0.0268 & $0.9845(3)$ & $0.0419(10)$ \\
\hline $\mathrm{C} 7$ & $0.4028(5)$ & -0.0460 & $0.9110(3)$ & $0.0445(11)$ \\
\hline $\mathrm{H} 7$ & 0.4717 & -0.0896 & 0.9164 & $0.053^{*}$ \\
\hline $\mathrm{C} 8$ & $0.3847(5)$ & $-0.0026(4)$ & $0.8321(3)$ & $0.0420(10)$ \\
\hline $\mathrm{H} 8$ & 0.4413 & -0.0143 & 0.7822 & $0.050^{*}$ \\
\hline C9 & $0.2804(4)$ & $0.0601(4)$ & $0.8257(3)$ & $0.0380(9)$ \\
\hline $\mathrm{C} 10$ & $0.2615(4)$ & $0.1096(3)$ & $0.7413(3)$ & $0.0363(9)$ \\
\hline H10 & 0.3216 & 0.0952 & 0.6939 & $0.044 *$ \\
\hline C11 & $-0.0066(4)$ & $0.2541(4)$ & $0.7732(3)$ & $0.0362(9)$ \\
\hline $\mathrm{C} 12$ & $-0.0996(4)$ & $0.2757(4)$ & $0.8476(3)$ & $0.0383(10)$ \\
\hline $\mathrm{C} 13$ & $-0.2028(5)$ & $0.3326(4)$ & $0.8370(3)$ & 0.0449 (11) \\
\hline H13 & -0.2171 & 0.3587 & 0.7815 & $0.054^{*}$ \\
\hline C14 & $-0.2851(5)$ & $0.3510(5)$ & $0.9086(4)$ & $0.0523(12)$ \\
\hline H14 & -0.3570 & 0.3911 & 0.9039 & $0.063 *$ \\
\hline $\mathrm{C} 15$ & $-0.2605(5)$ & $0.3099(5)$ & $0.9873(4)$ & $0.0546(13)$ \\
\hline H15 & -0.3157 & 0.3214 & 1.0378 & $0.066^{*}$ \\
\hline $\mathrm{C} 16$ & $-0.1564(5)$ & $0.2522(4)$ & $0.9926(3)$ & $0.0508(12)$ \\
\hline H16 & -0.1416 & 0.2240 & 1.0476 & $0.061 *$ \\
\hline $\mathrm{C} 17$ & $0.3043(5)$ & $0.3965(4)$ & $0.6477(3)$ & $0.0462(11)$ \\
\hline H17 & 0.3087 & 0.3453 & 0.6970 & $0.055^{*}$ \\
\hline $\mathrm{C} 18$ & $0.3708(6)$ & $0.4873(5)$ & $0.6321(4)$ & $0.0636(16)$ \\
\hline H18 & 0.4204 & 0.4983 & 0.6699 & $0.076^{*}$ \\
\hline C19 & $0.3650(6)$ & $0.5618(5)$ & $0.5616(4)$ & $0.0606(15)$ \\
\hline H19 & 0.4093 & 0.6255 & 0.5506 & $0.073^{*}$ \\
\hline $\mathrm{C} 20$ & $0.2942(5)$ & $0.5436(4)$ & $0.5065(4)$ & $0.0505(12)$ \\
\hline $\mathrm{H} 20$ & 0.2909 & 0.5940 & 0.4565 & $0.061^{*}$ \\
\hline $\mathrm{C} 21$ & $0.2283(4)$ & $0.4512(4)$ & $0.5250(3)$ & $0.0383(9)$ \\
\hline $\mathrm{C} 22$ & $0.1449(4)$ & $0.4264(3)$ & $0.4740(3)$ & $0.0364(9)$ \\
\hline $\mathrm{C} 23$ & $0.1209(5)$ & $0.4921(4)$ & $0.4014(3)$ & 0.0464 (11) \\
\hline $\mathrm{H} 23$ & 0.1620 & 0.5577 & 0.3810 & $0.056^{*}$ \\
\hline $\mathrm{C} 24$ & $0.0392(5)$ & $0.4629(4)$ & 0.3597 (3) & $0.0494(12)$ \\
\hline $\mathrm{H} 24$ & 0.0254 & 0.5066 & 0.3089 & $0.059^{*}$ \\
\hline $\mathrm{C} 25$ & $-0.0240(5)$ & $0.3690(4)$ & $0.3916(3)$ & $0.0472(12)$ \\
\hline $\mathrm{H} 25$ & -0.0830 & 0.3478 & 0.3641 & $0.057 *$ \\
\hline $\mathrm{C} 26$ & $0.0011(5)$ & $0.3071(4)$ & $0.4642(3)$ & $0.0405(10)$ \\
\hline $\mathrm{H} 26$ & -0.0428 & 0.2430 & 0.4870 & $0.049^{*}$ \\
\hline $\mathrm{C} 27$ & $0.3784(4)$ & $0.2201(4)$ & $0.4883(3)$ & $0.0418(10)$ \\
\hline $\mathrm{H} 27$ & 0.3987 & 0.2876 & 0.4929 & $0.050 *$ \\
\hline $\mathrm{C} 28$ & $0.4621(5)$ & $0.1703(4)$ & $0.4364(4)$ & $0.0495(12)$ \\
\hline $\mathrm{H} 28$ & 0.5392 & 0.2032 & 0.4054 & $0.059^{*}$ \\
\hline $\mathrm{C} 29$ & $0.4349(5)$ & $0.0727(4)$ & $0.4290(3)$ & $0.0464(11)$ \\
\hline H29 & 0.4916 & 0.0380 & 0.3919 & $0.056^{*}$ \\
\hline
\end{tabular}




$\begin{array}{lllll}\text { C30 } & 0.3234(5) & 0.0257(4) & 0.4765(3) & 0.0415(10) \\ \text { H30 } & 0.3037 & -0.0428 & 0.4741 & 0.050^{*} \\ \text { C31 } & 0.2414(4) & 0.0790(3) & 0.5273(3) & 0.0329(9) \\ \text { C32 } & 0.1175(4) & 0.0400(3) & 0.5761(3) & 0.0337(9) \\ \text { C33 } & 0.0747(5) & -0.0597(4) & 0.5847(3) & 0.0415(10) \\ \text { H33 } & 0.1283 & -0.1095 & 0.5622 & 0.050^{*} \\ \text { C34 } & -0.0470(5) & -0.0855(4) & 0.6263(3) & 0.0437(11) \\ \text { H34 } & -0.0774 & -0.1542 & 0.6348 & 0.052^{*} \\ \text { C35 } & -0.1245(4) & -0.0112(4) & 0.6554(3) & 0.0410(10) \\ \text { H35 } & -0.2102 & -0.0267 & 0.6805 & 0.049^{*} \\ \text { C36 } & -0.0763(4) & 0.0853(4) & 0.6478(3) & 0.0370(9) \\ \text { H36 } & -0.1293 & 0.1360 & 0.6693 & 0.044^{*} \\ \text { C37 } & 0.3274(11) & 0.3094(8) & 0.9504(7) & 0.106(3) \\ \text { H37A } & 0.3329 & 0.2505 & 0.9978 & 0.127^{*} \\ \text { H37B } & 0.2392 & 0.3155 & 0.9460 & 0.127^{*} \\ \text { C38 } & 0.0773(10) & 0.5757(8) & 0.8701(6) & 0.104(3) \\ \text { H38A } & 0.1078 & 0.5725 & 0.9228 & 0.125^{*} \\ \text { H38B } & 0.1143 & 0.6386 & 0.8284 & 0.125^{*} \\ \text { C39 } & 0.4343(9) & 0.3132(9) & 0.2089(7) & 0.115(3) \\ \text { H39A } & 0.5017 & 0.2796 & 0.2351 & 0.138^{*} \\ \text { H39B } & 0.4738 & 0.3558 & 0.1524 & 0.138^{*} \\ \text { B1 } & 0.6282(6) & 0.2076(5) & 0.6370(4) & 0.0492(13) \\ \text { B2 } & 0.7686(6) & 0.1829(5) & 0.2559(4) & 0.0522(14) \\ \text { H1 } & 0.093(5) & 0.165(4) & 0.835(4) & 0.043^{*} \\ & & & & \end{array}$

Atomic displacement parameters $\left(\AA^{2}\right)$

\begin{tabular}{lllllll}
\hline & $U^{11}$ & $U^{22}$ & $U^{33}$ & $U^{12}$ & $U^{13}$ & $U^{23}$ \\
\hline Ru1 & $0.0285(2)$ & $0.0341(2)$ & $0.03044(19)$ & $-0.00236(14)$ & $-0.00396(14)$ & $-0.00737(14)$ \\
C11 & $0.0815(15)$ & $0.1179(19)$ & $0.158(2)$ & $-0.0026(13)$ & $-0.0007(15)$ & $-0.0754(18)$ \\
C12 & $0.1014(16)$ & $0.0982(15)$ & $0.1238(18)$ & $0.0138(13)$ & $-0.0511(15)$ & $-0.0491(13)$ \\
Cl3 & $0.098(2)$ & $0.242(5)$ & $0.222(5)$ & $0.018(3)$ & $-0.008(3)$ & $-0.103(4)$ \\
C14 & $0.144(3)$ & $0.151(3)$ & $0.171(3)$ & $0.030(2)$ & $-0.078(2)$ & $-0.087(2)$ \\
C15 & $0.161(3)$ & $0.189(3)$ & $0.132(2)$ & $0.082(3)$ & $-0.038(2)$ & $-0.073(2)$ \\
C16 & $0.128(2)$ & $0.119(2)$ & $0.162(3)$ & $-0.0202(19)$ & $-0.008(2)$ & $-0.010(2)$ \\
F1 & $0.058(2)$ & $0.112(4)$ & $0.116(4)$ & $0.025(2)$ & $-0.033(3)$ & $-0.021(3)$ \\
F2 & $0.095(3)$ & $0.091(3)$ & $0.088(3)$ & $-0.012(2)$ & $-0.041(3)$ & $-0.034(2)$ \\
F3 & $0.078(3)$ & $0.057(2)$ & $0.073(2)$ & $-0.0024(19)$ & $0.007(2)$ & $0.0039(18)$ \\
F4 & $0.089(3)$ & $0.057(2)$ & $0.062(2)$ & $-0.0085(19)$ & $0.0078(19)$ & $-0.0097(16)$ \\
F5 & $0.083(3)$ & $0.091(3)$ & $0.073(2)$ & $0.005(2)$ & $-0.037(2)$ & $-0.007(2)$ \\
F6 & $0.151(5)$ & $0.073(3)$ & $0.086(3)$ & $-0.035(3)$ & $-0.056(3)$ & $-0.010(2)$ \\
F7 & $0.063(2)$ & $0.070(2)$ & $0.059(2)$ & $-0.0186(18)$ & $0.0040(17)$ & $-0.0126(17)$ \\
F8 & $0.053(2)$ & $0.157(5)$ & $0.080(3)$ & $0.013(3)$ & $0.012(2)$ & $-0.005(3)$ \\
O1 & $0.0318(15)$ & $0.0429(17)$ & $0.0311(14)$ & $-0.0014(13)$ & $-0.0034(12)$ & $-0.0099(12)$ \\
N1 & $0.036(2)$ & $0.049(2)$ & $0.0353(18)$ & $0.0017(17)$ & $-0.0058(16)$ & $-0.0083(16)$ \\
N2 & $0.0312(18)$ & $0.039(2)$ & $0.0313(17)$ & $-0.0015(15)$ & $-0.0015(15)$ & $-0.0081(15)$ \\
N3 & $0.036(2)$ & $0.048(2)$ & $0.0317(18)$ & $0.0078(17)$ & $-0.0050(16)$ & $-0.0075(17)$ \\
N4 & $0.045(2)$ & $0.052(2)$ & $0.039(2)$ & $0.0017(19)$ & $-0.0054(18)$ & $-0.0112(18)$
\end{tabular}




\begin{tabular}{|c|c|c|c|c|c|c|}
\hline N5 & $0.0287(18)$ & $0.0363(19)$ & $0.0356(18)$ & $0.0000(15)$ & $-0.0026(15)$ & $-0.0098(15)$ \\
\hline N6 & $0.0325(19)$ & 0.0349 (19) & $0.0321(17)$ & $-0.0021(15)$ & $-0.0044(15)$ & $-0.0081(14)$ \\
\hline N7 & $0.0290(18)$ & $0.040(2)$ & 0.0335 (17) & $-0.0039(15)$ & $-0.0030(15)$ & $-0.0107(15)$ \\
\hline N8 & $0.0304(18)$ & $0.0337(18)$ & $0.0292(16)$ & $0.0000(14)$ & $-0.0049(14)$ & $-0.0042(14)$ \\
\hline $\mathrm{C} 1$ & $0.038(2)$ & $0.043(3)$ & $0.039(2)$ & $-0.003(2)$ & $-0.008(2)$ & $-0.0024(19)$ \\
\hline $\mathrm{C} 2$ & $0.045(3)$ & $0.059(3)$ & $0.040(2)$ & $0.003(2)$ & -0.005 (2) & $-0.005(2)$ \\
\hline $\mathrm{C} 3$ & 0.055 (3) & $0.068(4)$ & $0.040(3)$ & $-0.002(3)$ & $-0.006(2)$ & $-0.009(2)$ \\
\hline $\mathrm{C} 4$ & 0.055 (3) & $0.069(4)$ & $0.036(2)$ & $-0.008(3)$ & $-0.015(2)$ & $0.001(2)$ \\
\hline $\mathrm{C} 5$ & $0.046(3)$ & 0.049 (3) & 0.045 & $-0.003(2)$ & $-0.019(2)$ & $-0.002(2)$ \\
\hline C6 & 0.037 (2) & 0.045 & $0.044(2)$ & $-0.007(2)$ & $-0.012(2)$ & $-0.004(2)$ \\
\hline $\mathrm{C} 7$ & 0.037 (2) & $0.046(3)$ & $0.052(3)$ & $0.003(2)$ & $-0.014(2)$ & $-0.009(2)$ \\
\hline $\mathrm{C} 8$ & 0.037 (2) & 0.048 & $0.039(2)$ & $0.001(2)$ & $-0.006(2)$ & $-0.009(2)$ \\
\hline C9 & 0.035 (2) & $0.039(2)$ & $0.038(2)$ & $-0.0033(19)$ & $-0.0049(19)$ & $-0.0068(18)$ \\
\hline $\mathrm{C} 10$ & $0.030(2)$ & $0.041(2)$ & $0.036(2)$ & $-0.0007(18)$ & $-0.0043(18)$ & $-0.0088(18)$ \\
\hline $\mathrm{C} 11$ & $0.032(2)$ & $0.039(2)$ & $0.037(2)$ & $-0.0015(18)$ & $-0.0025(18)$ & $-0.0122(18)$ \\
\hline C12 & $0.034(2)$ & $0.043(2)$ & $0.037(2)$ & -0.0055 (19) & $-0.0028(19)$ & $-0.0114(19)$ \\
\hline $\mathrm{C} 13$ & $0.038(3)$ & $0.050(3)$ & $0.044(2)$ & $0.005(2)$ & $-0.004(2)$ & $-0.009(2)$ \\
\hline $\mathrm{C} 14$ & $0.042(3)$ & $0.061(3)$ & $0.053(3)$ & $0.008(2)$ & $-0.004(2)$ & -0.018 \\
\hline $\mathrm{C} 15$ & 0.047 (3) & $0.064(3)$ & $0.049(3)$ & $0.003(3)$ & $0.005(2)$ & -0.021 \\
\hline $\mathrm{C} 16$ & $0.053(3)$ & $0.061(3)$ & $0.036(2)$ & $-0.002(3)$ & $-0.003(2)$ & -0.014 (2) \\
\hline $\mathrm{C} 17$ & $0.042(3)$ & $0.050(3)$ & 0.049 (3) & $-0.004(2)$ & $-0.011(2)$ & $-0.014(2)$ \\
\hline $\mathrm{C} 18$ & $0.056(3)$ & $0.066(4)$ & $0.079(4)$ & $-0.017(3)$ & $-0.023(3)$ & $-0.025(3)$ \\
\hline $\mathrm{C} 19$ & $0.052(3)$ & $0.052(3)$ & $0.079(4)$ & -0.019 & $-0.012(3)$ & $-0.016(3)$ \\
\hline $\mathrm{C} 20$ & $0.041(3)$ & $0.044(3)$ & $0.060(3)$ & $-0.010(2)$ & $-0.003(2)$ & $-0.003(2)$ \\
\hline $\mathrm{C} 21$ & $0.031(2)$ & $0.038(2)$ & $0.043(2)$ & $-0.0027(18)$ & $-0.0013(19)$ & $-0.0098(19)$ \\
\hline $\mathrm{C} 22$ & $0.033(2)$ & $0.036(2)$ & $0.036(2)$ & $0.0003(18)$ & $0.0014(18)$ & $-0.0081(18)$ \\
\hline $\mathrm{C} 23$ & $0.055(3)$ & $0.038(2)$ & $0.041(2)$ & $-0.002(2)$ & $-0.003(2)$ & $-0.0031(19)$ \\
\hline $\mathrm{C} 24$ & $0.055(3)$ & $0.046(3)$ & $0.043(3)$ & $0.005(2)$ & $-0.011(2)$ & $-0.002(2)$ \\
\hline $\mathrm{C} 25$ & $0.051(3)$ & $0.050(3)$ & $0.047(3)$ & $0.005(2)$ & $-0.020(2)$ & $-0.014(2)$ \\
\hline $\mathrm{C} 26$ & $0.041(3)$ & $0.041(2)$ & $0.040(2)$ & $-0.002(2)$ & $-0.010(2)$ & $-0.0094(19)$ \\
\hline $\mathrm{C} 27$ & $0.030(2)$ & $0.048(3)$ & $0.046(2)$ & $-0.006(2)$ & $-0.0010(19)$ & $-0.013(2)$ \\
\hline $\mathrm{C} 28$ & $0.032(2)$ & $0.058(3)$ & $0.055(3)$ & $-0.007(2)$ & $0.002(2)$ & $-0.016(2)$ \\
\hline $\mathrm{C} 29$ & $0.036(2)$ & $0.058(3)$ & $0.047(3)$ & $0.003(2)$ & $-0.002(2)$ & $-0.023(2)$ \\
\hline $\mathrm{C} 30$ & $0.038(2)$ & $0.044(3)$ & $0.045(2)$ & $0.001(2)$ & $-0.009(2)$ & $-0.015(2)$ \\
\hline C31 & $0.032(2)$ & $0.036(2)$ & $0.033(2)$ & $0.0001(17)$ & $-0.0099(17)$ & $-0.0092(17)$ \\
\hline $\mathrm{C} 32$ & $0.032(2)$ & $0.035(2)$ & $0.034(2)$ & $-0.0029(18)$ & $-0.0084(17)$ & $-0.0051(16)$ \\
\hline $\mathrm{C} 33$ & $0.042(3)$ & $0.040(2)$ & $0.044(2)$ & $0.000(2)$ & $-0.009(2)$ & $-0.0115(19)$ \\
\hline C34 & $0.045(3)$ & $0.038(2)$ & $0.045(2)$ & $-0.011(2)$ & $-0.007(2)$ & $-0.0057(19)$ \\
\hline $\mathrm{C} 35$ & $0.033(2)$ & $0.046(3)$ & $0.041(2)$ & $-0.009(2)$ & $-0.0024(19)$ & $-0.006(2)$ \\
\hline $\mathrm{C} 36$ & $0.030(2)$ & $0.043(2)$ & $0.035(2)$ & $-0.0026(19)$ & $-0.0031(18)$ & $-0.0073(18)$ \\
\hline C37 & $0.111(8)$ & $0.100(7)$ & $0.106(7)$ & $-0.020(6)$ & $-0.017(6)$ & $-0.026(5)$ \\
\hline C38 & $0.105(7)$ & $0.101(6)$ & $0.095(6)$ & $-0.039(6)$ & $0.006(5)$ & $-0.022(5)$ \\
\hline C39 & $0.070(6)$ & $0.151(9)$ & $0.121(8)$ & $0.012(6)$ & $0.004(5)$ & $-0.051(7)$ \\
\hline B1 & $0.043(3)$ & $0.054(3)$ & $0.050(3)$ & $-0.003(3)$ & $-0.009(3)$ & $-0.011(3)$ \\
\hline B2 & $0.045(3)$ & 0.059 (4) & 0.051 & $-0.004(3)$ & $-0.011(3)$ & -0.008 \\
\hline
\end{tabular}


Geometric parameters $\left(\AA,{ }^{\circ}\right)$

\begin{tabular}{|c|c|c|c|}
\hline $\mathrm{Ru} 1-\mathrm{N} 7$ & $2.036(4)$ & $\mathrm{C} 9-\mathrm{C} 10$ & $1.461(6)$ \\
\hline $\mathrm{Ru} 1-\mathrm{N} 2$ & $2.047(4)$ & $\mathrm{C} 10-\mathrm{H} 10$ & 0.9500 \\
\hline $\mathrm{Ru} 1-\mathrm{N} 8$ & $2.049(4)$ & $\mathrm{C} 11-\mathrm{C} 12$ & $1.480(6)$ \\
\hline $\mathrm{Ru} 1-\mathrm{N} 5$ & $2.054(4)$ & $\mathrm{C} 12-\mathrm{C} 13$ & $1.370(7)$ \\
\hline $\mathrm{Ru} 1-\mathrm{N} 6$ & $2.056(4)$ & $\mathrm{C} 13-\mathrm{C} 14$ & $1.376(7)$ \\
\hline $\mathrm{Ru} 1-\mathrm{O} 1$ & $2.090(3)$ & $\mathrm{C} 13-\mathrm{H} 13$ & 0.9500 \\
\hline $\mathrm{C} 11-\mathrm{C} 37$ & $1.770(9)$ & $\mathrm{C} 14-\mathrm{C} 15$ & $1.377(8)$ \\
\hline $\mathrm{Cl} 2-\mathrm{C} 37$ & $1.755(10)$ & C14-H14 & 0.9500 \\
\hline $\mathrm{Cl} 3-\mathrm{C} 38$ & $1.654(11)$ & $\mathrm{C} 15-\mathrm{C} 16$ & $1.369(8)$ \\
\hline $\mathrm{C} 14-\mathrm{C} 38$ & $1.700(10)$ & C15-H15 & 0.9500 \\
\hline $\mathrm{C} 15-\mathrm{C} 39$ & $1.688(10)$ & $\mathrm{C} 16-\mathrm{H} 16$ & 0.9500 \\
\hline $\mathrm{C} 16-\mathrm{C} 39$ & $1.786(11)$ & $\mathrm{C} 17-\mathrm{C} 18$ & $1.374(8)$ \\
\hline $\mathrm{F} 1-\mathrm{B} 1$ & $1.351(8)$ & $\mathrm{C} 17-\mathrm{H} 17$ & 0.9500 \\
\hline $\mathrm{F} 2-\mathrm{B} 1$ & $1.371(7)$ & $\mathrm{C} 18-\mathrm{C} 19$ & $1.365(9)$ \\
\hline $\mathrm{F} 3-\mathrm{B} 1$ & $1.369(8)$ & $\mathrm{C} 18-\mathrm{H} 18$ & 0.9500 \\
\hline $\mathrm{F} 4-\mathrm{B} 1$ & $1.399(7)$ & $\mathrm{C} 19-\mathrm{C} 20$ & $1.382(8)$ \\
\hline $\mathrm{F} 5-\mathrm{B} 2$ & $1.372(7)$ & C19-H19 & 0.9500 \\
\hline $\mathrm{F} 6-\mathrm{B} 2$ & $1.371(8)$ & $\mathrm{C} 20-\mathrm{C} 21$ & $1.383(7)$ \\
\hline $\mathrm{F} 7-\mathrm{B} 2$ & $1.370(7)$ & $\mathrm{C} 20-\mathrm{H} 20$ & 0.9500 \\
\hline $\mathrm{F} 8-\mathrm{B} 2$ & $1.370(8)$ & $\mathrm{C} 21-\mathrm{C} 22$ & $1.460(6)$ \\
\hline $\mathrm{O} 1-\mathrm{C} 11$ & $1.248(5)$ & $\mathrm{C} 22-\mathrm{C} 23$ & $1.394(7)$ \\
\hline N1-C9 & $1.322(6)$ & $\mathrm{C} 23-\mathrm{C} 24$ & $1.358(8)$ \\
\hline $\mathrm{N} 1-\mathrm{C} 1$ & $1.362(6)$ & $\mathrm{C} 23-\mathrm{H} 23$ & 0.9500 \\
\hline $\mathrm{N} 2-\mathrm{C} 10$ & $1.287(6)$ & $\mathrm{C} 24-\mathrm{C} 25$ & $1.385(8)$ \\
\hline $\mathrm{N} 2-\mathrm{N} 3$ & $1.386(5)$ & $\mathrm{C} 24-\mathrm{H} 24$ & 0.9500 \\
\hline N3-C11 & $1.321(6)$ & $\mathrm{C} 25-\mathrm{C} 26$ & $1.377(7)$ \\
\hline N3-H1 & $0.76(6)$ & $\mathrm{C} 25-\mathrm{H} 25$ & 0.9500 \\
\hline $\mathrm{N} 4-\mathrm{C} 16$ & $1.330(7)$ & $\mathrm{C} 26-\mathrm{H} 26$ & 0.9500 \\
\hline $\mathrm{N} 4-\mathrm{C} 12$ & $1.350(6)$ & $\mathrm{C} 27-\mathrm{C} 28$ & $1.364(7)$ \\
\hline $\mathrm{N} 5-\mathrm{C} 17$ & $1.338(6)$ & $\mathrm{C} 27-\mathrm{H} 27$ & 0.9500 \\
\hline $\mathrm{N} 5-\mathrm{C} 21$ & $1.367(6)$ & $\mathrm{C} 28-\mathrm{C} 29$ & $1.373(7)$ \\
\hline $\mathrm{N} 6-\mathrm{C} 26$ & $1.337(6)$ & $\mathrm{C} 28-\mathrm{H} 28$ & 0.9500 \\
\hline N6-C22 & $1.356(6)$ & $\mathrm{C} 29-\mathrm{C} 30$ & $1.382(7)$ \\
\hline N7-C27 & $1.335(6)$ & $\mathrm{C} 29-\mathrm{H} 29$ & 0.9500 \\
\hline N7-C31 & $1.368(5)$ & $\mathrm{C} 30-\mathrm{C} 31$ & $1.372(6)$ \\
\hline $\mathrm{N} 8-\mathrm{C} 36$ & $1.345(6)$ & $\mathrm{C} 30-\mathrm{H} 30$ & 0.9500 \\
\hline $\mathrm{N} 8-\mathrm{C} 32$ & $1.351(6)$ & $\mathrm{C} 31-\mathrm{C} 32$ & $1.464(6)$ \\
\hline $\mathrm{C} 1-\mathrm{C} 2$ & $1.407(7)$ & $\mathrm{C} 32-\mathrm{C} 33$ & $1.383(6)$ \\
\hline $\mathrm{C} 1-\mathrm{C} 6$ & $1.417(7)$ & $\mathrm{C} 33-\mathrm{C} 34$ & $1.376(7)$ \\
\hline $\mathrm{C} 2-\mathrm{C} 3$ & $1.367(7)$ & С $33-\mathrm{H} 33$ & 0.9500 \\
\hline $\mathrm{C} 2-\mathrm{H} 2$ & 0.9500 & $\mathrm{C} 34-\mathrm{C} 35$ & $1.375(7)$ \\
\hline $\mathrm{C} 3-\mathrm{C} 4$ & $1.412(8)$ & C $34-\mathrm{H} 34$ & 0.9500 \\
\hline $\mathrm{C} 3-\mathrm{H} 3$ & 0.9500 & $\mathrm{C} 35-\mathrm{C} 36$ & $1.368(6)$ \\
\hline $\mathrm{C} 4-\mathrm{C} 5$ & $1.344(8)$ & $\mathrm{C} 35-\mathrm{H} 35$ & 0.9500 \\
\hline $\mathrm{C} 4-\mathrm{H} 4$ & 0.9500 & $\mathrm{C} 36-\mathrm{H} 36$ & 0.9500 \\
\hline $\mathrm{C} 5-\mathrm{C} 6$ & $1.416(6)$ & $\mathrm{C} 37-\mathrm{H} 37 \mathrm{~A}$ & 0.9900 \\
\hline
\end{tabular}




\begin{tabular}{|c|c|c|c|}
\hline $\mathrm{C} 5-\mathrm{H} 5$ & 0.9500 & C37-H37B & 0.9900 \\
\hline $\mathrm{C} 6-\mathrm{C} 7$ & $1.400(7)$ & $\mathrm{C} 38-\mathrm{H} 38 \mathrm{~A}$ & 0.9900 \\
\hline $\mathrm{C} 7-\mathrm{C} 8$ & $1.359(7)$ & C $38-\mathrm{H} 38 \mathrm{~B}$ & 0.9900 \\
\hline $\mathrm{C} 7-\mathrm{H} 7$ & 0.9500 & C39-H39A & 0.9900 \\
\hline $\mathrm{C} 8-\mathrm{C} 9$ & $1.409(7)$ & C39-H39B & 0.9900 \\
\hline $\mathrm{C} 8-\mathrm{H} 8$ & 0.9500 & & \\
\hline $\mathrm{N} 7-\mathrm{Ru} 1-\mathrm{N} 2$ & $96.41(15)$ & $\mathrm{C} 19-\mathrm{C} 18-\mathrm{C} 17$ & $119.4(5)$ \\
\hline $\mathrm{N} 7-\mathrm{Ru} 1-\mathrm{N} 8$ & $79.11(14)$ & $\mathrm{C} 19-\mathrm{C} 18-\mathrm{H} 18$ & 120.3 \\
\hline $\mathrm{N} 2-\mathrm{Ru} 1-\mathrm{N} 8$ & $87.08(14)$ & $\mathrm{C} 17-\mathrm{C} 18-\mathrm{H} 18$ & 120.3 \\
\hline N7-Ru1-N5 & $95.80(14)$ & $\mathrm{C} 18-\mathrm{C} 19-\mathrm{C} 20$ & $119.4(5)$ \\
\hline $\mathrm{N} 2-\mathrm{Ru} 1-\mathrm{N} 5$ & $96.69(14)$ & $\mathrm{C} 18-\mathrm{C} 19-\mathrm{H} 19$ & 120.3 \\
\hline $\mathrm{N} 8-\mathrm{Ru} 1-\mathrm{N} 5$ & $174.01(13)$ & $\mathrm{C} 20-\mathrm{C} 19-\mathrm{H} 19$ & 120.3 \\
\hline $\mathrm{N} 7-\mathrm{Ru} 1-\mathrm{N} 6$ & $90.26(15)$ & $\mathrm{C} 19-\mathrm{C} 20-\mathrm{C} 21$ & $119.2(5)$ \\
\hline $\mathrm{N} 2-\mathrm{Ru} 1-\mathrm{N} 6$ & $172.50(14)$ & $\mathrm{C} 19-\mathrm{C} 20-\mathrm{H} 20$ & 120.4 \\
\hline $\mathrm{N} 8-\mathrm{Ru} 1-\mathrm{N} 6$ & $97.59(14)$ & $\mathrm{C} 21-\mathrm{C} 20-\mathrm{H} 20$ & 120.4 \\
\hline N5-Ru1-N6 & 79.15 (14) & $\mathrm{N} 5-\mathrm{C} 21-\mathrm{C} 20$ & $121.0(4)$ \\
\hline $\mathrm{N} 7-\mathrm{Ru} 1-\mathrm{O} 1$ & $172.56(13)$ & $\mathrm{N} 5-\mathrm{C} 21-\mathrm{C} 22$ & $115.1(4)$ \\
\hline $\mathrm{N} 2-\mathrm{Ru} 1-\mathrm{O} 1$ & $78.77(13)$ & $\mathrm{C} 20-\mathrm{C} 21-\mathrm{C} 22$ & $123.8(5)$ \\
\hline $\mathrm{N} 8-\mathrm{Ru} 1-\mathrm{O} 1$ & $94.89(13)$ & $\mathrm{N} 6-\mathrm{C} 22-\mathrm{C} 23$ & $120.0(4)$ \\
\hline $\mathrm{N} 5-\mathrm{Ru} 1-\mathrm{O} 1$ & $90.42(13)$ & $\mathrm{N} 6-\mathrm{C} 22-\mathrm{C} 21$ & $115.2(4)$ \\
\hline $\mathrm{N} 6-\mathrm{Ru} 1-\mathrm{O} 1$ & $94.92(13)$ & $\mathrm{C} 23-\mathrm{C} 22-\mathrm{C} 21$ & $124.8(4)$ \\
\hline $\mathrm{C} 11-\mathrm{O} 1-\mathrm{Ru} 1$ & $111.9(3)$ & $\mathrm{C} 24-\mathrm{C} 23-\mathrm{C} 22$ & $120.3(5)$ \\
\hline $\mathrm{C} 9-\mathrm{N} 1-\mathrm{C} 1$ & $119.3(4)$ & $\mathrm{C} 24-\mathrm{C} 23-\mathrm{H} 23$ & 119.9 \\
\hline $\mathrm{C} 10-\mathrm{N} 2-\mathrm{N} 3$ & $117.3(4)$ & $\mathrm{C} 22-\mathrm{C} 23-\mathrm{H} 23$ & 119.9 \\
\hline $\mathrm{C} 10-\mathrm{N} 2-\mathrm{Ru} 1$ & $132.9(3)$ & $\mathrm{C} 23-\mathrm{C} 24-\mathrm{C} 25$ & $119.6(5)$ \\
\hline N3-N2-Ru1 & $109.9(3)$ & $\mathrm{C} 23-\mathrm{C} 24-\mathrm{H} 24$ & 120.2 \\
\hline $\mathrm{C} 11-\mathrm{N} 3-\mathrm{N} 2$ & $117.9(4)$ & $\mathrm{C} 25-\mathrm{C} 24-\mathrm{H} 24$ & 120.2 \\
\hline $\mathrm{C} 11-\mathrm{N} 3-\mathrm{H} 1$ & $128(4)$ & $\mathrm{C} 26-\mathrm{C} 25-\mathrm{C} 24$ & $118.2(5)$ \\
\hline N2-N3-H1 & $114(4)$ & $\mathrm{C} 26-\mathrm{C} 25-\mathrm{H} 25$ & 120.9 \\
\hline $\mathrm{C} 16-\mathrm{N} 4-\mathrm{C} 12$ & $116.4(5)$ & $\mathrm{C} 24-\mathrm{C} 25-\mathrm{H} 25$ & 120.9 \\
\hline $\mathrm{C} 17-\mathrm{N} 5-\mathrm{C} 21$ & $118.5(4)$ & $\mathrm{N} 6-\mathrm{C} 26-\mathrm{C} 25$ & $122.7(5)$ \\
\hline $\mathrm{C} 17-\mathrm{N} 5-\mathrm{Ru} 1$ & $126.5(3)$ & $\mathrm{N} 6-\mathrm{C} 26-\mathrm{H} 26$ & 118.6 \\
\hline $\mathrm{C} 21-\mathrm{N} 5-\mathrm{Ru} 1$ & $114.9(3)$ & $\mathrm{C} 25-\mathrm{C} 26-\mathrm{H} 26$ & 118.6 \\
\hline $\mathrm{C} 26-\mathrm{N} 6-\mathrm{C} 22$ & $119.2(4)$ & $\mathrm{N} 7-\mathrm{C} 27-\mathrm{C} 28$ & $122.1(4)$ \\
\hline $\mathrm{C} 26-\mathrm{N} 6-\mathrm{Ru} 1$ & $125.6(3)$ & $\mathrm{N} 7-\mathrm{C} 27-\mathrm{H} 27$ & 118.9 \\
\hline $\mathrm{C} 22-\mathrm{N} 6-\mathrm{Ru} 1$ & $115.2(3)$ & $\mathrm{C} 28-\mathrm{C} 27-\mathrm{H} 27$ & 118.9 \\
\hline $\mathrm{C} 27-\mathrm{N} 7-\mathrm{C} 31$ & $118.5(4)$ & $\mathrm{C} 27-\mathrm{C} 28-\mathrm{C} 29$ & $119.9(5)$ \\
\hline $\mathrm{C} 27-\mathrm{N} 7-\mathrm{Ru} 1$ & $126.1(3)$ & $\mathrm{C} 27-\mathrm{C} 28-\mathrm{H} 28$ & 120.0 \\
\hline $\mathrm{C} 31-\mathrm{N} 7-\mathrm{Ru} 1$ & $115.3(3)$ & $\mathrm{C} 29-\mathrm{C} 28-\mathrm{H} 28$ & 120.0 \\
\hline $\mathrm{C} 36-\mathrm{N} 8-\mathrm{C} 32$ & $119.0(4)$ & $\mathrm{C} 28-\mathrm{C} 29-\mathrm{C} 30$ & $118.8(5)$ \\
\hline $\mathrm{C} 36-\mathrm{N} 8-\mathrm{Ru} 1$ & $125.9(3)$ & $\mathrm{C} 28-\mathrm{C} 29-\mathrm{H} 29$ & 120.6 \\
\hline $\mathrm{C} 32-\mathrm{N} 8-\mathrm{Ru} 1$ & $114.9(3)$ & $\mathrm{C} 30-\mathrm{C} 29-\mathrm{H} 29$ & 120.6 \\
\hline $\mathrm{N} 1-\mathrm{C} 1-\mathrm{C} 2$ & $118.6(5)$ & $\mathrm{C} 31-\mathrm{C} 30-\mathrm{C} 29$ & $119.3(4)$ \\
\hline $\mathrm{N} 1-\mathrm{C} 1-\mathrm{C} 6$ & $121.4(4)$ & $\mathrm{C} 31-\mathrm{C} 30-\mathrm{H} 30$ & 120.4 \\
\hline $\mathrm{C} 2-\mathrm{C} 1-\mathrm{C} 6$ & $120.0(4)$ & $\mathrm{C} 29-\mathrm{C} 30-\mathrm{H} 30$ & 120.4 \\
\hline $\mathrm{C} 3-\mathrm{C} 2-\mathrm{C} 1$ & $120.0(5)$ & $\mathrm{N} 7-\mathrm{C} 31-\mathrm{C} 30$ & $121.3(4)$ \\
\hline $\mathrm{C} 3-\mathrm{C} 2-\mathrm{H} 2$ & 120.0 & $\mathrm{~N} 7-\mathrm{C} 31-\mathrm{C} 32$ & $114.4(4)$ \\
\hline
\end{tabular}




\begin{tabular}{|c|c|c|c|}
\hline $\mathrm{C} 1-\mathrm{C} 2-\mathrm{H} 2$ & 120.0 & $\mathrm{C} 30-\mathrm{C} 31-\mathrm{C} 32$ & $124.2(4)$ \\
\hline $\mathrm{C} 2-\mathrm{C} 3-\mathrm{C} 4$ & $119.8(5)$ & $\mathrm{N} 8-\mathrm{C} 32-\mathrm{C} 33$ & $121.2(4)$ \\
\hline $\mathrm{C} 2-\mathrm{C} 3-\mathrm{H} 3$ & 120.1 & $\mathrm{~N} 8-\mathrm{C} 32-\mathrm{C} 31$ & $114.8(4)$ \\
\hline $\mathrm{C} 4-\mathrm{C} 3-\mathrm{H} 3$ & 120.1 & $\mathrm{C} 33-\mathrm{C} 32-\mathrm{C} 31$ & $124.0(4)$ \\
\hline $\mathrm{C} 5-\mathrm{C} 4-\mathrm{C} 3$ & $121.2(5)$ & $\mathrm{C} 34-\mathrm{C} 33-\mathrm{C} 32$ & $118.9(5)$ \\
\hline $\mathrm{C} 5-\mathrm{C} 4-\mathrm{H} 4$ & 119.4 & C $34-\mathrm{C} 33-\mathrm{H} 33$ & 120.6 \\
\hline $\mathrm{C} 3-\mathrm{C} 4-\mathrm{H} 4$ & 119.4 & $\mathrm{C} 32-\mathrm{C} 33-\mathrm{H} 33$ & 120.6 \\
\hline $\mathrm{C} 4-\mathrm{C} 5-\mathrm{C} 6$ & $120.7(5)$ & $\mathrm{C} 35-\mathrm{C} 34-\mathrm{C} 33$ & $119.6(4)$ \\
\hline $\mathrm{C} 4-\mathrm{C} 5-\mathrm{H} 5$ & 119.6 & $\mathrm{C} 35-\mathrm{C} 34-\mathrm{H} 34$ & 120.2 \\
\hline $\mathrm{C} 6-\mathrm{C} 5-\mathrm{H} 5$ & 119.6 & C33-C34-H34 & 120.2 \\
\hline $\mathrm{C} 7-\mathrm{C} 6-\mathrm{C} 5$ & $124.4(5)$ & $\mathrm{C} 36-\mathrm{C} 35-\mathrm{C} 34$ & $119.0(4)$ \\
\hline $\mathrm{C} 7-\mathrm{C} 6-\mathrm{C} 1$ & $117.5(4)$ & $\mathrm{C} 36-\mathrm{C} 35-\mathrm{H} 35$ & 120.5 \\
\hline $\mathrm{C} 5-\mathrm{C} 6-\mathrm{C} 1$ & $118.1(5)$ & C34-C35-H35 & 120.5 \\
\hline $\mathrm{C} 8-\mathrm{C} 7-\mathrm{C} 6$ & $120.5(5)$ & $\mathrm{N} 8-\mathrm{C} 36-\mathrm{C} 35$ & $122.0(4)$ \\
\hline $\mathrm{C} 8-\mathrm{C} 7-\mathrm{H} 7$ & 119.7 & $\mathrm{~N} 8-\mathrm{C} 36-\mathrm{H} 36$ & 119.0 \\
\hline $\mathrm{C} 6-\mathrm{C} 7-\mathrm{H} 7$ & 119.7 & $\mathrm{C} 35-\mathrm{C} 36-\mathrm{H} 36$ & 119.0 \\
\hline $\mathrm{C} 7-\mathrm{C} 8-\mathrm{C} 9$ & $118.8(4)$ & $\mathrm{Cl} 2-\mathrm{C} 37-\mathrm{Cl} 1$ & $111.0(6)$ \\
\hline $\mathrm{C} 7-\mathrm{C} 8-\mathrm{H} 8$ & 120.6 & $\mathrm{Cl} 2-\mathrm{C} 37-\mathrm{H} 37 \mathrm{~A}$ & 109.4 \\
\hline $\mathrm{C} 9-\mathrm{C} 8-\mathrm{H} 8$ & 120.6 & $\mathrm{Cl1}-\mathrm{C} 37-\mathrm{H} 37 \mathrm{~A}$ & 109.4 \\
\hline $\mathrm{N} 1-\mathrm{C} 9-\mathrm{C} 8$ & $122.5(4)$ & $\mathrm{Cl} 2-\mathrm{C} 37-\mathrm{H} 37 \mathrm{~B}$ & 109.4 \\
\hline $\mathrm{N} 1-\mathrm{C} 9-\mathrm{C} 10$ & $118.1(4)$ & $\mathrm{C} 11-\mathrm{C} 37-\mathrm{H} 37 \mathrm{~B}$ & 109.4 \\
\hline $\mathrm{C} 8-\mathrm{C} 9-\mathrm{C} 10$ & $119.4(4)$ & $\mathrm{H} 37 \mathrm{~A}-\mathrm{C} 37-\mathrm{H} 37 \mathrm{~B}$ & 108.0 \\
\hline $\mathrm{N} 2-\mathrm{C} 10-\mathrm{C} 9$ & $128.1(4)$ & $\mathrm{Cl} 3-\mathrm{C} 38-\mathrm{Cl} 4$ & $113.1(6)$ \\
\hline $\mathrm{N} 2-\mathrm{C} 10-\mathrm{H} 10$ & 115.9 & $\mathrm{Cl} 3-\mathrm{C} 38-\mathrm{H} 38 \mathrm{~A}$ & 109.0 \\
\hline $\mathrm{C} 9-\mathrm{C} 10-\mathrm{H} 10$ & 115.9 & $\mathrm{Cl} 4-\mathrm{C} 38-\mathrm{H} 38 \mathrm{~A}$ & 109.0 \\
\hline $\mathrm{O} 1-\mathrm{C} 11-\mathrm{N} 3$ & $121.4(4)$ & $\mathrm{Cl} 3-\mathrm{C} 38-\mathrm{H} 38 \mathrm{~B}$ & 109.0 \\
\hline $\mathrm{O} 1-\mathrm{C} 11-\mathrm{C} 12$ & $122.6(4)$ & $\mathrm{Cl} 4-\mathrm{C} 38-\mathrm{H} 38 \mathrm{~B}$ & 109.0 \\
\hline $\mathrm{N} 3-\mathrm{C} 11-\mathrm{C} 12$ & $116.0(4)$ & $\mathrm{H} 38 \mathrm{~A}-\mathrm{C} 38-\mathrm{H} 38 \mathrm{~B}$ & 107.8 \\
\hline $\mathrm{N} 4-\mathrm{C} 12-\mathrm{C} 13$ & $124.1(4)$ & $\mathrm{Cl} 5-\mathrm{C} 39-\mathrm{Cl} 6$ & $114.6(6)$ \\
\hline $\mathrm{N} 4-\mathrm{C} 12-\mathrm{C} 11$ & $114.9(4)$ & $\mathrm{Cl} 5-\mathrm{C} 39-\mathrm{H} 39 \mathrm{~A}$ & 108.6 \\
\hline $\mathrm{C} 13-\mathrm{C} 12-\mathrm{C} 11$ & $121.0(4)$ & $\mathrm{Cl} 6-\mathrm{C} 39-\mathrm{H} 39 \mathrm{~A}$ & 108.6 \\
\hline $\mathrm{C} 12-\mathrm{C} 13-\mathrm{C} 14$ & $118.3(5)$ & $\mathrm{Cl} 5-\mathrm{C} 39-\mathrm{H} 39 \mathrm{~B}$ & 108.6 \\
\hline $\mathrm{C} 12-\mathrm{C} 13-\mathrm{H} 13$ & 120.9 & $\mathrm{Cl} 6-\mathrm{C} 39-\mathrm{H} 39 \mathrm{~B}$ & 108.6 \\
\hline $\mathrm{C} 14-\mathrm{C} 13-\mathrm{H} 13$ & 120.9 & H39A-C39-H39B & 107.6 \\
\hline $\mathrm{C} 13-\mathrm{C} 14-\mathrm{C} 15$ & $118.3(5)$ & $\mathrm{F} 1-\mathrm{B} 1-\mathrm{F} 3$ & $113.2(5)$ \\
\hline $\mathrm{C} 13-\mathrm{C} 14-\mathrm{H} 14$ & 120.9 & $\mathrm{~F} 1-\mathrm{B} 1-\mathrm{F} 2$ & $111.3(5)$ \\
\hline $\mathrm{C} 15-\mathrm{C} 14-\mathrm{H} 14$ & 120.9 & $\mathrm{~F} 3-\mathrm{B} 1-\mathrm{F} 2$ & $109.4(5)$ \\
\hline $\mathrm{C} 16-\mathrm{C} 15-\mathrm{C} 14$ & $119.9(5)$ & $\mathrm{F} 1-\mathrm{B} 1-\mathrm{F} 4$ & $108.2(5)$ \\
\hline $\mathrm{C} 16-\mathrm{C} 15-\mathrm{H} 15$ & 120.1 & $\mathrm{~F} 3-\mathrm{B} 1-\mathrm{F} 4$ & $108.2(5)$ \\
\hline $\mathrm{C} 14-\mathrm{C} 15-\mathrm{H} 15$ & 120.1 & $\mathrm{~F} 2-\mathrm{B} 1-\mathrm{F} 4$ & $106.3(5)$ \\
\hline $\mathrm{N} 4-\mathrm{C} 16-\mathrm{C} 15$ & $123.0(5)$ & $\mathrm{F} 8-\mathrm{B} 2-\mathrm{F} 7$ & $108.7(6)$ \\
\hline N4-C16-H16 & 118.5 & $\mathrm{~F} 8-\mathrm{B} 2-\mathrm{F} 6$ & $110.9(6)$ \\
\hline $\mathrm{C} 15-\mathrm{C} 16-\mathrm{H} 16$ & 118.5 & $\mathrm{~F} 7-\mathrm{B} 2-\mathrm{F} 6$ & $108.2(5)$ \\
\hline $\mathrm{N} 5-\mathrm{C} 17-\mathrm{C} 18$ & $122.5(5)$ & $\mathrm{F} 8-\mathrm{B} 2-\mathrm{F} 5$ & $108.1(5)$ \\
\hline N5-C17-H17 & 118.8 & F7-B2-F5 & $110.7(5)$ \\
\hline $\mathrm{C} 18-\mathrm{C} 17-\mathrm{H} 17$ & 118.8 & $\mathrm{~F} 6-\mathrm{B} 2-\mathrm{F} 5$ & $110.3(6)$ \\
\hline $\mathrm{C} 10-\mathrm{N} 2-\mathrm{N} 3-\mathrm{C} 11$ & $179.3(4)$ & $\mathrm{C} 17-\mathrm{N} 5-\mathrm{C} 21-\mathrm{C} 20$ & $-0.2(7)$ \\
\hline
\end{tabular}




\begin{tabular}{|c|c|c|c|}
\hline $\mathrm{Ru} 1-\mathrm{N} 2-\mathrm{N} 3-\mathrm{C} 11$ & $-1.9(5)$ & $\mathrm{Ru} 1-\mathrm{N} 5-\mathrm{C} 21-\mathrm{C} 20$ & $-178.0(4)$ \\
\hline $\mathrm{C} 9-\mathrm{N} 1-\mathrm{C} 1-\mathrm{C} 2$ & $179.0(5)$ & $\mathrm{C} 17-\mathrm{N} 5-\mathrm{C} 21-\mathrm{C} 22$ & $-177.6(4)$ \\
\hline $\mathrm{C} 9-\mathrm{N} 1-\mathrm{C} 1-\mathrm{C} 6$ & $0.8(7)$ & $\mathrm{Ru} 1-\mathrm{N} 5-\mathrm{C} 21-\mathrm{C} 22$ & $4.7(5)$ \\
\hline $\mathrm{N} 1-\mathrm{C} 1-\mathrm{C} 2-\mathrm{C} 3$ & $-178.0(5)$ & $\mathrm{C} 19-\mathrm{C} 20-\mathrm{C} 21-\mathrm{N} 5$ & $-0.8(8)$ \\
\hline $\mathrm{C} 6-\mathrm{C} 1-\mathrm{C} 2-\mathrm{C} 3$ & $0.2(8)$ & $\mathrm{C} 19-\mathrm{C} 20-\mathrm{C} 21-\mathrm{C} 22$ & $176.3(5)$ \\
\hline $\mathrm{C} 1-\mathrm{C} 2-\mathrm{C} 3-\mathrm{C} 4$ & $-0.2(9)$ & $\mathrm{C} 26-\mathrm{N} 6-\mathrm{C} 22-\mathrm{C} 23$ & $-1.2(7)$ \\
\hline $\mathrm{C} 2-\mathrm{C} 3-\mathrm{C} 4-\mathrm{C} 5$ & $-1.0(9)$ & $\mathrm{Ru} 1-\mathrm{N} 6-\mathrm{C} 22-\mathrm{C} 23$ & $178.5(4)$ \\
\hline $\mathrm{C} 3-\mathrm{C} 4-\mathrm{C} 5-\mathrm{C} 6$ & $2.2(8)$ & $\mathrm{C} 26-\mathrm{N} 6-\mathrm{C} 22-\mathrm{C} 21$ & $176.1(4)$ \\
\hline $\mathrm{C} 4-\mathrm{C} 5-\mathrm{C} 6-\mathrm{C} 7$ & $179.1(5)$ & $\mathrm{Ru} 1-\mathrm{N} 6-\mathrm{C} 22-\mathrm{C} 21$ & $-4.2(5)$ \\
\hline $\mathrm{C} 4-\mathrm{C} 5-\mathrm{C} 6-\mathrm{C} 1$ & $-2.2(7)$ & $\mathrm{N} 5-\mathrm{C} 21-\mathrm{C} 22-\mathrm{N} 6$ & $-0.3(6)$ \\
\hline $\mathrm{N} 1-\mathrm{C} 1-\mathrm{C} 6-\mathrm{C} 7$ & $-2.1(7)$ & $\mathrm{C} 20-\mathrm{C} 21-\mathrm{C} 22-\mathrm{N} 6$ & $-177.6(5)$ \\
\hline $\mathrm{C} 2-\mathrm{C} 1-\mathrm{C} 6-\mathrm{C} 7$ & $179.8(5)$ & $\mathrm{N} 5-\mathrm{C} 21-\mathrm{C} 22-\mathrm{C} 23$ & $176.8(4)$ \\
\hline $\mathrm{N} 1-\mathrm{C} 1-\mathrm{C} 6-\mathrm{C} 5$ & $179.1(4)$ & $\mathrm{C} 20-\mathrm{C} 21-\mathrm{C} 22-\mathrm{C} 23$ & $-0.4(8)$ \\
\hline $\mathrm{C} 2-\mathrm{C} 1-\mathrm{C} 6-\mathrm{C} 5$ & $1.0(7)$ & $\mathrm{N} 6-\mathrm{C} 22-\mathrm{C} 23-\mathrm{C} 24$ & $-1.2(7)$ \\
\hline $\mathrm{C} 5-\mathrm{C} 6-\mathrm{C} 7-\mathrm{C} 8$ & $-178.9(5)$ & $\mathrm{C} 21-\mathrm{C} 22-\mathrm{C} 23-\mathrm{C} 24$ & $-178.2(5)$ \\
\hline $\mathrm{C} 1-\mathrm{C} 6-\mathrm{C} 7-\mathrm{C} 8$ & $2.3(7)$ & $\mathrm{C} 22-\mathrm{C} 23-\mathrm{C} 24-\mathrm{C} 25$ & $2.5(8)$ \\
\hline $\mathrm{C} 6-\mathrm{C} 7-\mathrm{C} 8-\mathrm{C} 9$ & $-1.4(7)$ & $\mathrm{C} 23-\mathrm{C} 24-\mathrm{C} 25-\mathrm{C} 26$ & $-1.5(8)$ \\
\hline $\mathrm{C} 1-\mathrm{N} 1-\mathrm{C} 9-\mathrm{C} 8$ & $0.3(7)$ & $\mathrm{C} 22-\mathrm{N} 6-\mathrm{C} 26-\mathrm{C} 25$ & $2.3(7)$ \\
\hline $\mathrm{C} 1-\mathrm{N} 1-\mathrm{C} 9-\mathrm{C} 10$ & $-178.1(4)$ & $\mathrm{Ru} 1-\mathrm{N} 6-\mathrm{C} 26-\mathrm{C} 25$ & $-177.4(4)$ \\
\hline $\mathrm{C} 7-\mathrm{C} 8-\mathrm{C} 9-\mathrm{N} 1$ & $0.0(7)$ & $\mathrm{C} 24-\mathrm{C} 25-\mathrm{C} 26-\mathrm{N} 6$ & $-0.9(8)$ \\
\hline $\mathrm{C} 7-\mathrm{C} 8-\mathrm{C} 9-\mathrm{C} 10$ & $178.3(4)$ & $\mathrm{C} 31-\mathrm{N} 7-\mathrm{C} 27-\mathrm{C} 28$ & $1.3(7)$ \\
\hline $\mathrm{N} 3-\mathrm{N} 2-\mathrm{C} 10-\mathrm{C} 9$ & $-1.0(7)$ & $\mathrm{Ru} 1-\mathrm{N} 7-\mathrm{C} 27-\mathrm{C} 28$ & $-178.1(4)$ \\
\hline $\mathrm{Ru} 1-\mathrm{N} 2-\mathrm{C} 10-\mathrm{C} 9$ & $-179.4(3)$ & $\mathrm{N} 7-\mathrm{C} 27-\mathrm{C} 28-\mathrm{C} 29$ & $-0.2(8)$ \\
\hline $\mathrm{N} 1-\mathrm{C} 9-\mathrm{C} 10-\mathrm{N} 2$ & $-0.4(7)$ & $\mathrm{C} 27-\mathrm{C} 28-\mathrm{C} 29-\mathrm{C} 30$ & $-1.6(8)$ \\
\hline $\mathrm{C} 8-\mathrm{C} 9-\mathrm{C} 10-\mathrm{N} 2$ & $-178.8(5)$ & $\mathrm{C} 28-\mathrm{C} 29-\mathrm{C} 30-\mathrm{C} 31$ & $2.3(7)$ \\
\hline $\mathrm{Ru} 1-\mathrm{O} 1-\mathrm{C} 11-\mathrm{N} 3$ & $1.5(5)$ & $\mathrm{C} 27-\mathrm{N} 7-\mathrm{C} 31-\mathrm{C} 30$ & $-0.7(6)$ \\
\hline $\mathrm{Ru} 1-\mathrm{O} 1-\mathrm{C} 11-\mathrm{C} 12$ & $-179.3(3)$ & $\mathrm{Ru} 1-\mathrm{N} 7-\mathrm{C} 31-\mathrm{C} 30$ & $178.8(3)$ \\
\hline $\mathrm{N} 2-\mathrm{N} 3-\mathrm{C} 11-\mathrm{O} 1$ & $0.3(7)$ & $\mathrm{C} 27-\mathrm{N} 7-\mathrm{C} 31-\mathrm{C} 32$ & $-178.0(4)$ \\
\hline $\mathrm{N} 2-\mathrm{N} 3-\mathrm{C} 11-\mathrm{C} 12$ & $-179.0(4)$ & $\mathrm{Ru} 1-\mathrm{N} 7-\mathrm{C} 31-\mathrm{C} 32$ & $1.6(5)$ \\
\hline $\mathrm{C} 16-\mathrm{N} 4-\mathrm{C} 12-\mathrm{C} 13$ & $-1.3(7)$ & $\mathrm{C} 29-\mathrm{C} 30-\mathrm{C} 31-\mathrm{N} 7$ & $-1.1(7)$ \\
\hline $\mathrm{C} 16-\mathrm{N} 4-\mathrm{C} 12-\mathrm{C} 11$ & $179.7(4)$ & $\mathrm{C} 29-\mathrm{C} 30-\mathrm{C} 31-\mathrm{C} 32$ & $175.9(4)$ \\
\hline $\mathrm{O} 1-\mathrm{C} 11-\mathrm{C} 12-\mathrm{N} 4$ & $-174.6(4)$ & $\mathrm{C} 36-\mathrm{N} 8-\mathrm{C} 32-\mathrm{C} 33$ & $-5.6(6)$ \\
\hline $\mathrm{N} 3-\mathrm{C} 11-\mathrm{C} 12-\mathrm{N} 4$ & $4.6(6)$ & $\mathrm{Ru} 1-\mathrm{N} 8-\mathrm{C} 32-\mathrm{C} 33$ & $169.3(3)$ \\
\hline $\mathrm{O} 1-\mathrm{C} 11-\mathrm{C} 12-\mathrm{C} 13$ & $6.4(7)$ & $\mathrm{C} 36-\mathrm{N} 8-\mathrm{C} 32-\mathrm{C} 31$ & $172.1(4)$ \\
\hline $\mathrm{N} 3-\mathrm{C} 11-\mathrm{C} 12-\mathrm{C} 13$ & $-174.4(5)$ & $\mathrm{Ru} 1-\mathrm{N} 8-\mathrm{C} 32-\mathrm{C} 31$ & $-12.9(4)$ \\
\hline $\mathrm{N} 4-\mathrm{C} 12-\mathrm{C} 13-\mathrm{C} 14$ & $1.8(8)$ & $\mathrm{N} 7-\mathrm{C} 31-\mathrm{C} 32-\mathrm{N} 8$ & $7.5(5)$ \\
\hline $\mathrm{C} 11-\mathrm{C} 12-\mathrm{C} 13-\mathrm{C} 14$ & $-179.3(5)$ & $\mathrm{C} 30-\mathrm{C} 31-\mathrm{C} 32-\mathrm{N} 8$ & $-169.7(4)$ \\
\hline $\mathrm{C} 12-\mathrm{C} 13-\mathrm{C} 14-\mathrm{C} 15$ & $-1.0(8)$ & $\mathrm{N} 7-\mathrm{C} 31-\mathrm{C} 32-\mathrm{C} 33$ & $-174.8(4)$ \\
\hline $\mathrm{C} 13-\mathrm{C} 14-\mathrm{C} 15-\mathrm{C} 16$ & $-0.1(9)$ & $\mathrm{C} 30-\mathrm{C} 31-\mathrm{C} 32-\mathrm{C} 33$ & $8.0(7)$ \\
\hline $\mathrm{C} 12-\mathrm{N} 4-\mathrm{C} 16-\mathrm{C} 15$ & $0.1(8)$ & $\mathrm{N} 8-\mathrm{C} 32-\mathrm{C} 33-\mathrm{C} 34$ & $2.7(7)$ \\
\hline $\mathrm{C} 14-\mathrm{C} 15-\mathrm{C} 16-\mathrm{N} 4$ & $0.6(9)$ & $\mathrm{C} 31-\mathrm{C} 32-\mathrm{C} 33-\mathrm{C} 34$ & $-174.8(4)$ \\
\hline $\mathrm{C} 21-\mathrm{N} 5-\mathrm{C} 17-\mathrm{C} 18$ & $0.6(8)$ & $\mathrm{C} 32-\mathrm{C} 33-\mathrm{C} 34-\mathrm{C} 35$ & $2.4(7)$ \\
\hline $\mathrm{Ru} 1-\mathrm{N} 5-\mathrm{C} 17-\mathrm{C} 18$ & $178.0(4)$ & $\mathrm{C} 33-\mathrm{C} 34-\mathrm{C} 35-\mathrm{C} 36$ & $-4.6(7)$ \\
\hline $\mathrm{N} 5-\mathrm{C} 17-\mathrm{C} 18-\mathrm{C} 19$ & $0.2(9)$ & $\mathrm{C} 32-\mathrm{N} 8-\mathrm{C} 36-\mathrm{C} 35$ & $3.4(6)$ \\
\hline $\mathrm{C} 17-\mathrm{C} 18-\mathrm{C} 19-\mathrm{C} 20$ & $-1.2(10)$ & $\mathrm{Ru} 1-\mathrm{N} 8-\mathrm{C} 36-\mathrm{C} 35$ & $-170.9(3)$ \\
\hline $\mathrm{C} 18-\mathrm{C} 19-\mathrm{C} 20-\mathrm{C} 21$ & $1.5(9)$ & $\mathrm{C} 34-\mathrm{C} 35-\mathrm{C} 36-\mathrm{N} 8$ & $1.7(7)$ \\
\hline
\end{tabular}


supporting information

Hydrogen-bond geometry $\left(\AA,{ }^{\circ}\right)$

\begin{tabular}{lllll}
\hline$D-\mathrm{H} \cdots A$ & $D-\mathrm{H}$ & $\mathrm{H} \cdots A$ & $D \cdots A$ & $D-\mathrm{H} \cdots A$ \\
\hline $\mathrm{N} 3-\mathrm{H} 1 \cdots \mathrm{N} 1$ & $0.76(6)$ & $1.90(6)$ & $2.553(6)$ & $145(6)$ \\
\hline
\end{tabular}

